Research Paper

\title{
Age-related DNA methylation changes are sex-specific: a comprehensive assessment
}

Igor Yusipov ${ }^{1,2, *}$, Maria Giulia Bacalini ${ }^{3, *}$, Alena Kalyakulina ${ }^{1, *}$, Mikhail Krivonosov ${ }^{1}$, Chiara Pirazzini ${ }^{3}$, Noémie Gensous ${ }^{4}$, Francesco Ravaioli ${ }^{4}$, Maddalena Milazzo ${ }^{4}$, Cristina Giuliani ${ }^{5,6}$, Maria Vedunova $^{7}$, Giovanni Fiorito ${ }^{8,9}$, Amedeo Gagliardi ${ }^{10,11}$, Silvia Polidoro ${ }^{9,10,11}$, Paolo Garagnani $^{4,12,13,14}$, Mikhail Ivanchenko ${ }^{1,2, \#}$, Claudio Franceschi ${ }^{1, \#}$

${ }^{1}$ Institute of Information Technologies, Mathematics and Mechanics, Lobachevsky University, Nizhniy Novgorod, Russia

${ }^{2}$ Mathematics of Future Technologies Center, Lobachevsky University, Nizhniy Novgorod, Russia

${ }^{3}$ IRCCS Istituto delle Scienze Neurologiche di Bologna, Bologna, Italy

${ }^{4}$ Department of Experimental, Diagnostic and Specialty Medicine (DIMES), Alma Mater Studiorum - University of Bologna, Bologna, Italy

${ }^{5}$ Department of Biological, Geological, and Environmental Sciences (BiGeA), Laboratory of Molecular Anthropology and Centre for Genome Biology, University of Bologna, Bologna, Italy

${ }^{6}$ School of Anthropology and Museum Ethnography, University of Oxford, Oxford, UK

${ }^{7}$ Institute of Biology and Biomedicine, National Research Lobachevsky State University of Nizhni Novgorod, Nizhni Novgorod, Russia

${ }^{8}$ Department of Biomedical Sciences, University of Sassari, Italy

${ }^{9}$ Department of Epidemiology and Public Health, MRC/HPA Centre for Environment and Health, School of Public Health, Imperial College London, Norfolk Place, London W2 1PG, UK

${ }^{10}$ Italian Institute for Genomic Medicine (IIGM), Candiolo 10060, Italy

${ }^{11}$ Candiolo Cancer Institute, FPO-IRCCS, Candiolo 10060, Italy

${ }^{12}$ Department of Laboratory Medicine, Clinical Chemistry, Karolinska Institutet, Karolinska University Hospital, Stockholm, Sweden

${ }^{13}$ Applied Biomedical Research Center (CRBA), Policlinico S.Orsola-Malpighi Polyclinic, Bologna, Italy

${ }^{14}$ CNR Institute of Molecular Genetics "Luigi Luca Cavalli-Sforza", Unit of Bologna, Bologna, Italy

${ }^{*}$ Co-first authorship

"Co-senior authorship

Correspondence to: Maria Giulia Bacalini; email: mariagiulia.bacalini@ausl.bologna.it

Keywords: methylation, sex, meta-analysis, whole blood, variability

Abbreviations: sDMPs: sex-associated differentially methylated positions; aDMPs: age-associated differentially methylated positions; saDMPs: sex- and age-associated differentially methylated positions; snaDMPs: sex- but not age-associated differentially methylated positions; saVMPs: sex-specific age-associated variably methylated positions.

Received: July 30, $2020 \quad$ Accepted: October 19, $2020 \quad$ Published: December 3, 2020

Copyright: (c) 2020 Yusipov et al. This is an open access article distributed under the terms of the Creative Commons Attribution License (CC BY 3.0), which permits unrestricted use, distribution, and reproduction in any medium, provided the original author and source are credited.

\section{ABSTRACT}

The existence of a sex gap in human health and longevity has been widely documented. Autosomal DNA methylation differences between males and females have been reported, but so far, few studies have investigated if DNA methylation is differently affected by aging in males and females. We performed a metaanalysis of 4 large whole blood datasets, comparing 4 aspects of epigenetic age-dependent remodeling 
between the two sexes: differential methylation, variability, epimutations and entropy. We reported that a large fraction (43\%) of sex-associated probes undergoes age-associated DNA methylation changes, and that a limited number of probes show age-by-sex interaction. We experimentally validated 2 regions mapping in FIGN and PRR4 genes and showed sex-specific deviations of their methylation patterns in models of decelerated (centenarians) and accelerated (Down syndrome) aging. While we did not find sex differences in the ageassociated increase in epimutations and entropy, we showed that the number of probes having an age-related increase in methylation variability is $\mathbf{1 5}$ times higher in males compared to females. Our results can offer new epigenetic tools to study the interaction between aging and sex and can pave the way to the identification of molecular triggers of sex differences in longevity and age-related diseases prevalence.

\section{INTRODUCTION}

A profound and multifaceted remodeling of DNA methylation patterns occurs during human aging [1-3]. DNA methylation profiles tend to diverge among individuals during life course [4-6], shaped by an intricate combination of environmental exposures, random events and genetically-driven mechanisms. Several epigenome-wide association studies (EWAS) have shown that a subset of the about 28 million $\mathrm{CpG}$ sites of the genome undergoes age-associated normative changes, i.e. reproducible hypermethylation or hypomethylation events that normally occur in all individuals during physiological, healthy aging (normative aging) [7,8]. Despite some controversial results $[9,10]$, at least a fraction of normative epigenetic changes is tissue-specific, indicating that the cellular microenvironment affects the activity of the molecular writers of DNA methylation patterns during aging. In the last 10 years an increasing number of studies identified age-associated DNA methylation changes at the level of single $\mathrm{CpG}$ sites, paving the way for the development of models, termed "epigenetic clocks", that predict age starting from the epigenetic profile [11]. Epigenetic clocks are an appealing resource for chronological age estimation in forensic applications, but they have risen to the limelight particularly because multiple reports have shown that they are sensitive to health status and are thus informative of the biological age of an individual. Although a conclusive association between epigenetic clock predictions and risk of age-related diseases is still missing [12], several independent studies showed that epigenetic age acceleration (i.e., predicted epigenetic age higher than effective chronological age) is associated with age-related diseases like cancer, cardiovascular disease and neurodegenerative conditions and to all-cause mortality [13]. On the other side, epigenetic age deceleration was reported to be associated with successful aging and longevity $[14,15]$.

Surprisingly, the research on the DNA methylation changes occurring during aging has largely neglected one of the hot topics in aging research, i.e. the sex differences in lifespan and health span.

According to Global Health Observatory (GHO) data [16], global life expectancy at birth in 2016 was 74.2 years for females and 69.8 years for males and, although with a different extent, this sex gap in longevity is worldwide [17]. At the same time, epidemiological data indicate that women live longer than men but experience a worse quality of life in advanced age [18]. Sex disparity exists for several diseases: cardiovascular disease, cancer and Parkinson's disease have higher mortality rates in males than in females at a given age, while females are at higher risk of Alzheimer's disease and show an increased prevalence of disabling conditions like bone and joint problems and autoimmune diseases. The reasons for these differences are still unclear, but they likely result from a strict interplay between nature (for example, differences in hormone levels, asymmetries in genetic inheritance, sexual dimorphism) and nurture (for example, different vulnerability to environmental hazards, sexual selection) [19]. Notably, sex-specific longevity loci have been recently identified [20], further pointing out the contribution of sex on aging trajectories.

Independent studies reported DNA methylation differences between males and females in various tissues [21-23], involving $\mathrm{CpG}$ sites widespread across the autosomal chromosomes. These differences mirror the diversity in transcriptomic and proteomic profiles between the two sexes that have been recently reported $[24,25]$. However, few studies have investigated whether DNA methylation differences exist between males and females during aging and whether they contribute to the sex gap in aging and longevity. According to Horvath's clock, males have an acceleration in epigenetic age compared to females [26-28]. Masser et al. analyzed genome-wide DNA methylation in mouse hippocampus and human frontal cortex and reported $\mathrm{CpG}$ sites that show different DNA methylation levels between males and females lifelong (referred as sex differences) and $\mathrm{CpG}$ sites that are 
differently affected by aging in males and females (referred as sex divergence) [29]. The vast majority of EWAS studies on aging have been performed in whole blood, but sex has usually been exiled as a confounding factor and used to adjust DNA methylation data.

In the present work we specifically investigated sex differences in whole blood DNA methylation changes during aging. We provide the results of a comprehensive study of 4 large whole blood datasets considering different aspects of age-associated epigenetic remodeling that can, either individually or in combination, contribute to the sex-specificity of human aging and longevity. In particular we focused on: 1) age-related changes in DNA methylation levels $[8,30]$; 2 ) age-related increase in DNA methylation variability, as described by Slieker et al. [6]; 3) age-related increase in epimutations, i.e. rare or stochastic changes in DNA methylation levels that are not shared among subjects, as defined by Gentilini et al. [5] 4) age-related increase in entropy in DNA methylation profiles, as previously described by Hannum et al. [31]. Furthermore, we investigated a subset of the loci emerged from these analyses in human models of successful and unsuccessful aging. Our results are compared with the only study that, to the best of our knowledge, recently investigated age-by-sex DNA methylation differences in whole blood of a single population, i.e. a large Scottish cohort [32].

\section{RESULTS}

\section{Identification of sex- and age-associated differentially methylated positions}

We performed a meta-analysis of 4 large datasets on whole blood (Materials and Methods) to identify $\mathrm{CpG}$ sites with differential methylation between males and females (sex-associated differentially methylated positions, sDMPs). We identified 38100 sDMPs (Bonferroni corrected $\mathrm{p}$-values resulting from metaanalysis $<0.01$ ), $53 \%$ of which were hypermethylated in females compared to males. We used the same datasets to identify age-associated differentially methylated positions (aDMPs) and we selected a list of 87581 probes (Bonferroni corrected p-values resulting from meta-analysis <0.01), 52\% of which underwent hypermethylation with aging. We then asked how many sDMPs underwent DNA methylation changes with age, i.e. were also aDMPs. The intersection between sDMPs and aDMPs lists returned 16526 probes, that we defined sex- and age-associated differentially methylated positions (saDMPs); we defined the remaining probes (21574) sex- but not age-associated differentially methylated positions (snaDMPs). Figure 1A reports a graphical representation of the procedure used to identify saDMPs and snaDMPs. The proportion of sexassociated probes showing age-associated changes (16526 out 38100) was higher then expected, considering the proportion of age-associated probes in the genome (87581 out 327905; Fisher's exact test pvalue $<2 * 10-16$, odds ratio 2.35 ). We found that most of the saDMPs undergoing hypomethylation with age were more methylated in males compared to females, while most of the age-hypermethylated saDMPs were more methylated in females compared to males (Figure 1B). Four examples of saDMPs are depicted in Figure 1C. The lists of saDMPs and snaDMPs are reported in Supplementary File 1.

When compared to previously published studies, we found that a total of 1121 saDMPs and 2163 snaDMPs were reported to have sex-dependent methylation (independently from age) in previous reports [21-23], also when newborns were considered [23] (Supplementary File 1).

We then investigated the possible functional role of saDMPs and snaDMPs. First of all, we explored whether the selected probes were enriched in specific genomic regions (Supplementary Figure 1), and we found that both saDMPs and snaDMPs were significantly enriched in Shore regions. The list of saDMPs, but not of snaDMPs, was significantly depleted in imprinted regions ( $\mathrm{p}$-value $=0.04$, odds ratio: 0.64), as defined by Court et al. [33]. We also checked for the presence of sex hormone-related genes, as suggested by [22], in the lists of saDMPs and snaDMPs. saDMPs and snaDMPs mapped in 6610 and 8367 genes respectively. 2899 genes were shared between the two lists, indicating that the same gene can include multiple $\mathrm{CpG}$ sites with DNA methylation differences between males and females, only a subset of which shows also age-associated changes. The list of saDMPs included a higher proportion of hormonerelated genes (28/6610 genes for saDMPs; 29/8367 for snaDMPs), but the enrichment was not statistically significant (p-value: 0.08; odds ratio: 1.6). Then, we analyzed the two lists for their enrichment in gene ontologies according to the GO database. While the list of snaDMPs was not enriched in any biological process (FDR corrected $\mathrm{p}$-value $<0.01$ ), saDMPs were enriched in multiple ontologies related to neuronal and developmental functions and to cell-cell interactions (Supplementary File 2).

Finally, we evaluated age-by-sex interactions in the 4 datasets. Meta-analysis resulted in $8 \mathrm{CpG}$ probes whose methylation showed different aging trajectories according to sex (Supplementary File 3). Two of these $\mathrm{CpG}$ sites were previously identified as having age-bysex interaction in whole blood [32], and the most 
significant CpG site (cg18834375) mapped within the FIGN gene, that was the top ranker also in the list of saDMPs and included multiple CpG sites showing sexand age-dependent methylation (cg01620164, cg19156483, cg10864319, cg18834375, cg15259986 and $\operatorname{cg} 03878133)$.

\section{Validation of saDMPs}

A subset of the above-identified saDMPs was experimentally validated using the EpiTYPER assay, a high throughput approach for target DNA methylation analysis. Target regions were chosen within FIGN and PRR4. We analyzed whole blood from 198 males from 15 to 98 years old and 221 females from 23 to 98 years old.

The FIGN target region included $13 \mathrm{CpG}$ sites; of these, 7 were measurable by the assay, grouped in $5 \mathrm{CpG}$ units. $\mathrm{CpG}$ unit 3.4.5 included the microarray probe $\operatorname{cg} 01620164$. We found that this group of $\mathrm{CpG}$ sites showed a sex-specific DNA hypomethylation trajectory comparable to what observed in the microarray (Supplementary Figure 2); also, the adjacent $\mathrm{CpG}$ sites showed a similar profile (Supplementary Figure 2), in particular CpG unit 9 (Figure 2A). This result indicates that the $\mathrm{CpG}$ sites located in this genomic region of at least $250 \mathrm{bp}$ are concordantly regulated in whole blood during aging according to sex.

The PRR4 target region included $5 \mathrm{CpG}$ sites, all assessable by EpiTYPER and all corresponding to an Infinium450k probe; $\mathrm{CpG}$ units 3 and 4, corresponding to the Infinium450k probes $\operatorname{cg} 23256579$ and cg27615582, had the same mass and returned the same methylation value in the EpiTYPER assay. While $\mathrm{CpG}$ units 1 and 2 did not show age-dependent changes nor sex specificity (Supplementary Figure 3), CpG units 3 and 4 showed sex-dependent trajectories with aging (Figure 2B). Although less evident, also CpG 5 showed sex-related differences in age-associated methylation changes (Supplementary Figure 3).
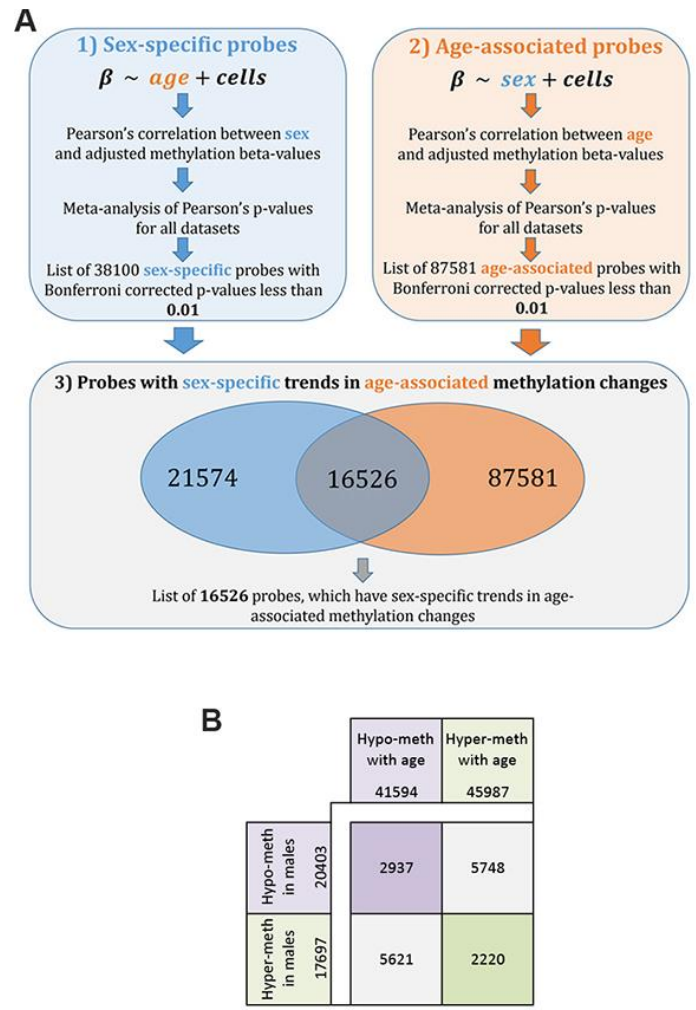

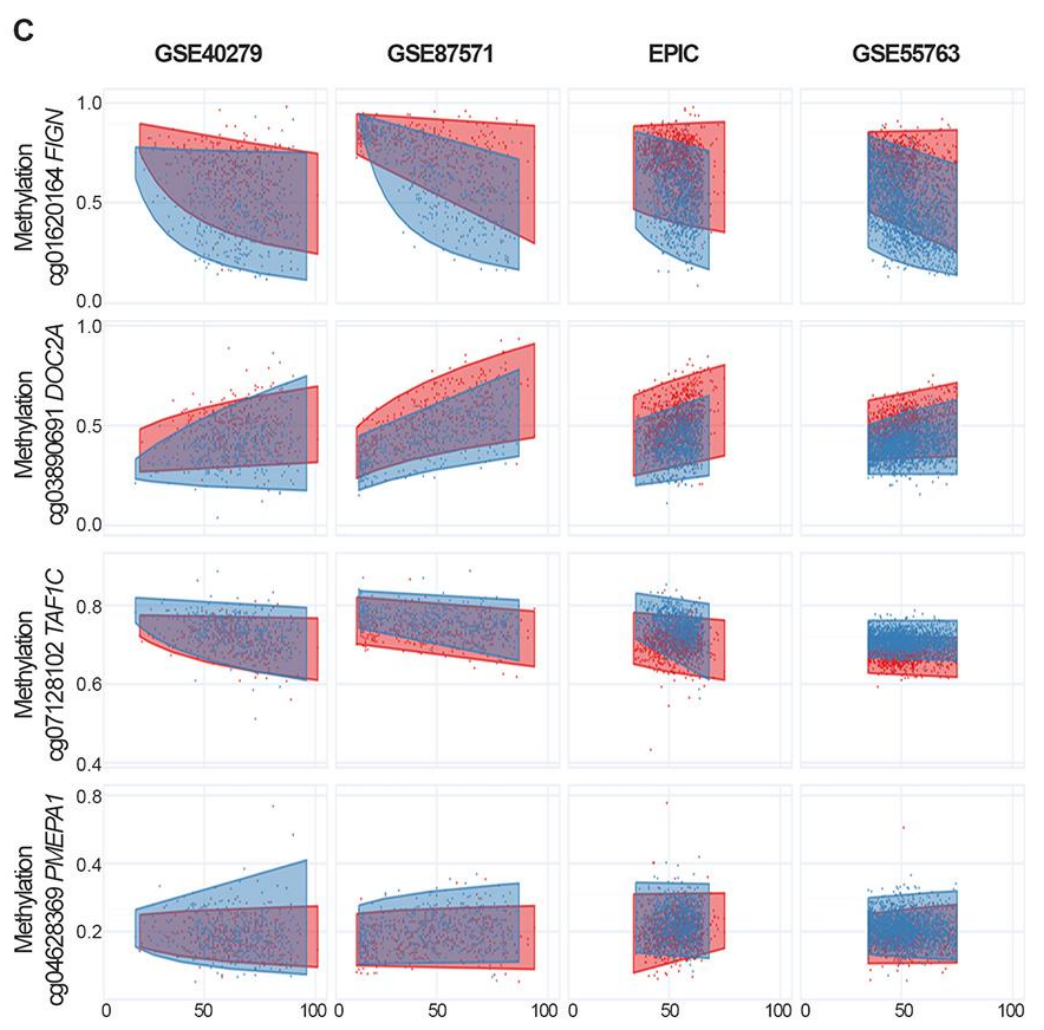

Figure 1. Identification of sex- and age-associated differentially methylated positions (saDMPs). (A) The procedure used to identify saDMPs and snaDMPs. (B) Scheme of the number of SDMPs, aDMPs and saDMPs, divided according to the direction of methylation changes respect to sex (hyper- or hypo-methylated in males compared to females) and age (hyper-or hypo-methylated with increasing age). (C) Scatter plots of a selection of saDMPs: cg01620164 is hypomethylated in males and un dergoes age-associated hypomethylation; cg03890691 is hypomethylated in males and undergoes age-associated hypermethylation; cg07128102 is hypermethylated in males and undergoes age-associated hypomethylation; cg04628369 is hypermethylated in males and undergoes age-associated hypermethylation. 
We used the EpiTYPER assay to investigate the two validated loci in samples from additional cohorts available in our laboratory: persons affected by Down syndrome, that we previously demonstrated to have an acceleration in epigenetic age $[34,35]$; and centenarians and their offspring, as a model of successful aging experiencing a deceleration in epigenetic age [14]. Interestingly, we found sex-dependent patterns of FIGN and PRR4 methylation also in these models. Compared to aged controls (>80 years old), centenarian males displayed highly variable DNA methylation profiles for FIGN amplicon, with about half of the subjects showing a female-like DNA methylation level (Figure 2A, 2C, Supplementary Figure 4); the differences in variance between control and centenarians' males (but not females) reached statistical significance for $\mathrm{CpG}$ unit 9 (F-test p-value: 0.02). No specific trends were found for PRR4 amplicon in the centenarians' cohort (Supplementary Figure 5). Centenarians' offspring showed DNA methylation patterns comparable to age-matched controls for both the amplicons
(Supplementary Figures 4 and 5). Persons affected by Down syndrome showed DNA methylation profiles similar to age-matched controls in FIGN locus (Supplementary Figure 4). On the contrary, females affected by Down syndrome showed lower values of CpG unit 3 in PRR4 amplicon compared to sex- and age-matched healthy controls (ANOVA p-value correcting for age: $6.2 * 10-5$ ), while no significant differences were found between males affected by Down syndrome compared to sex- and age-matched controls (Figure 2B and 2D, Supplementary Figure 5). The results of the statistical analyses performed on the centenarians', centenarians' offspring and Down syndrome cohorts are summarized in Supplementary File 4.

\section{Identification of sex-specific age-associated variably methylated positions (saVMPs)}

An increase in inter-individual DNA methylation variability has been described during aging, but possible
A
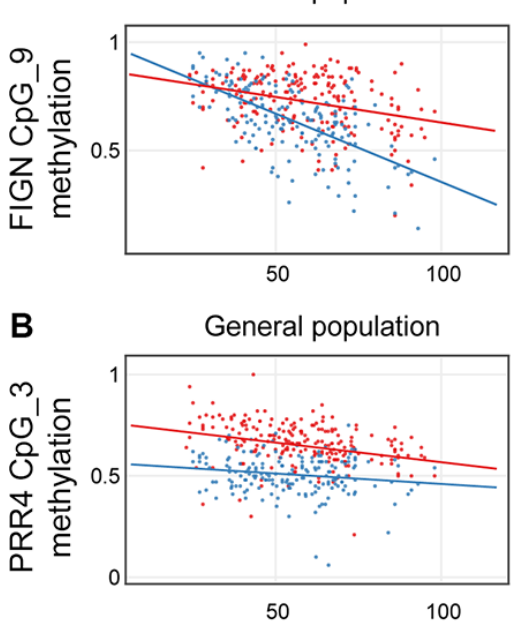

C

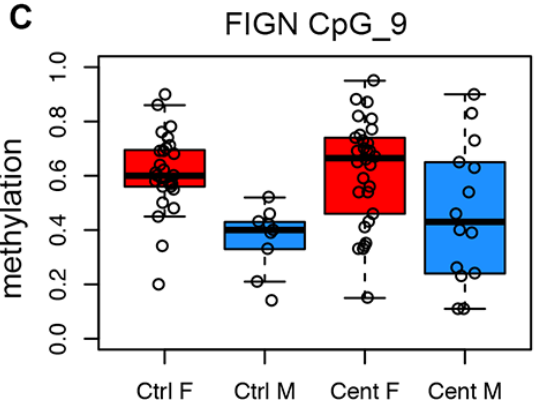

Centenarians' offspring

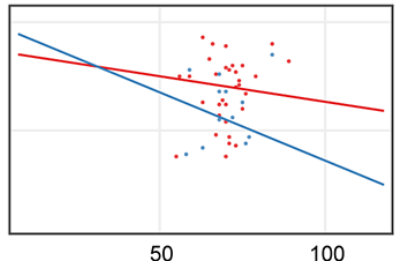

Centenarians' offspring

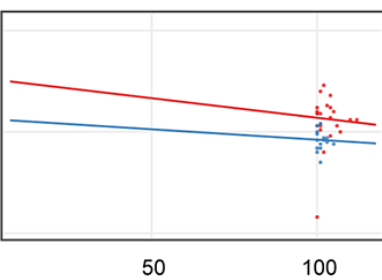

Persons with Down Syndrome

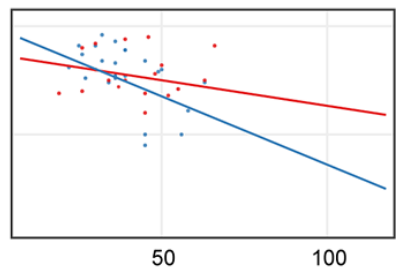

Persons with Down Syndrome

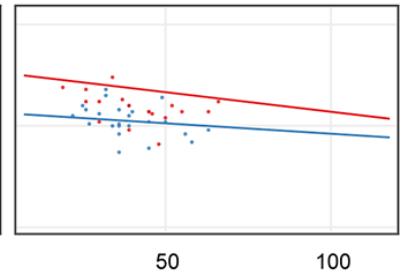

D

PRR4 CPG_3

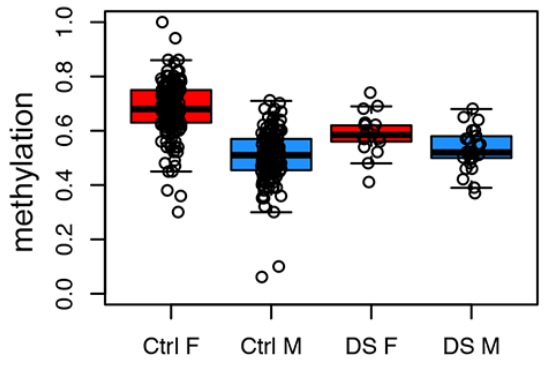

Figure 2. Validation of FIGN and PRR4 loci by EpiTYPER. (A) Methylation of CpG unit 9 in FIGN amplicon vs age. (B) Methylation of CpG unit 3 in PRR4 amplicon vs age. (A and B) For each CpG unit, DNA methylation in controls (general population), centenarians, centenarian's offspring and persons with Down syndrome is reported vs the age of the subjects. Males are in blue, females are in red. Linear regression between DNA methylation and age was calculated separately for males and females in control subjects and was reported in each plot. (C) Boxplots of DNA methylation of CpG unit 9 in FIGN amplicon in aged controls and centenarians. (D) Boxplots of DNA methylation of CpG unit 3 in PRR4 amplicon in healthy controls and persons with Down syndrome. 
sex-specific effects have not been investigated so far. To have a general view of the sex-dependent trends in age-related increase in DNA methylation variability, we plotted the density distributions of standard deviation values, calculated in the GSE87571 dataset (the one with the most homogeneous distribution of ages) in 3 age-ranges (14-39 years; 40-59 years; 60-94 years) considering the whole cohort (Figure 3A) or separating males and females (Figure 3B). A clear increase in standard deviation across the 3 age ranges was evident when considering the entire cohort. No clear differences between males and females were evident in the first 2 age ranges, while we found a trend towards higher variability in males in the oldest group.

To identify probes having sex-specific differences in age-dependent variability (sex-specific age-associated variably methylated positions, saVMPs), we applied the approach described in Materials and Methods and reported in Figure 4A. We identified 809 and 12178 saVMPs specific for females and males respectively (Supplementary File 5). All the female-specific saVMPs displayed increased variability with age, and similarly only for 5 out of 12178 male-specific saVMPs variability decreased with age. No probes with opposite trends in the two sexes were identified. Some examples of female- and male-specific saVMPs are reported in Figures 4B-4E.

While female-specific saVMPs were enriched in Islands, male-specific saVMPs were enriched in Shore regions (Supplementary Figure 6). We also found that male-specific saVMPs were enriched in imprinted regions and mapped in 20 hormone-related genes, although this enrichment was not significant (p-value: 0.09; odds ratio: 1.66). Both female- and male-specific
saVMPs were enriched in several gene ontologies related to neuronal and developmental processes, with some ontologies shared between the two lists (Supplementary File 6).

\section{Epimutations and entropy analysis}

Epimutations were calculated in each dataset as previously described [5]. As shown in Figure 5A, we confirmed an increase in the number of epimutations with age both in males and females (p-value $<0.01$ in all the datasets), but no sex-specific trends were found according to ANCOVA model (Supplementary Table 1).

The dependence of Shannon entropy on age for the 4 datasets is shown in Figure 5B. Entropy showed a significant increase with age (p-value $<0.01)$ in the GSE87571, EPIC and GSE55763 datasets (Supplementary Table 1), while it differed between the two sexes only in the EPIC dataset. EPIC dataset. These results suggest that there were no robust differences between sexes in Shannon entropy age-dependent increase.

\section{DISCUSSION}

Males and females experience different aging trajectories for several phenotypic traits [36, 37]. Sexspecificity is established and maintained by differential genomic regulation, as evidenced by the profound transcriptomic, epigenomic and proteomic differences between males and females. However, how these differences in genome regulation evolve during life course has been poorly investigated, thus leaving a gap in our understanding of sexual dimorphism in aging and of its consequences in terms of morbidity and mortality.
B

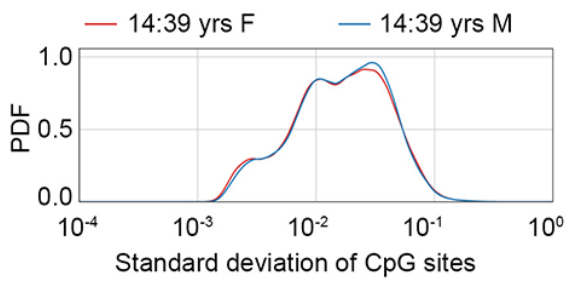

A
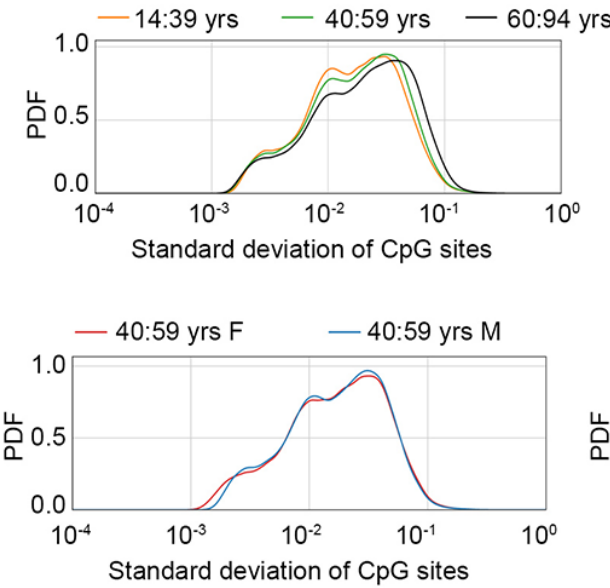

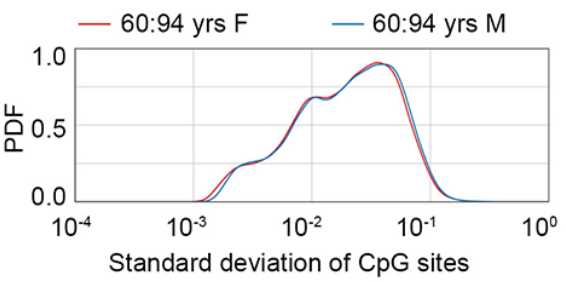

Figure 3. Probability density function (PDF) of standard deviation values calculated in the GSE87571 dataset for 3 age classes, considering males and females together (A) or separated (B). 
In the present study we aimed at filling this gap by exploiting 4 large EWAS on human whole blood, including men and women of different ages and populations, in which we analyzed the sex specificity of age-associated normative changes, variability, epimutations and entropy.

The main findings we will discuss are the following: i) a large fraction of probes with sex-specific DNA methylation undergoes also hyper- or hypo-methylation during aging, and a small number of probes show significant age-by-sex interaction; ii) the methylation of 2 selected saDMPs, mapping in FIGN and PRR4 genes, is differently modulated in centenarians and Down syndrome persons, assumed as human models of successful and unsuccessful aging [14, 34, 35]; iii) males display a higher number of saVMPs respect to females, the vast majority of which show an ageassociated increase in methylation variability; iv) males and females do not differ for the age-associated increase in epimutations and entropy.

\section{saDMPs in healthy subjects of different ages and populations.}

We reported that $43 \%$ of probes showing sex-associated DNA methylation differences in whole blood display also age-associated changes (saDMPs). This result suggests that $\mathrm{CpG}$ sites with blood DNA methylation differences between males and females are particularly prone to undergo epigenetic changes during aging. Interestingly we found that while saDMPs were enriched in gene ontologies related to neuronal and developmental functions, snaDMPs were not enriched in any particular biological process. When considering autosomal differences in DNA methylation between men and women (correcting for age), Singmann et al. found an enrichment in $\mathrm{CpG}$ island shores and in

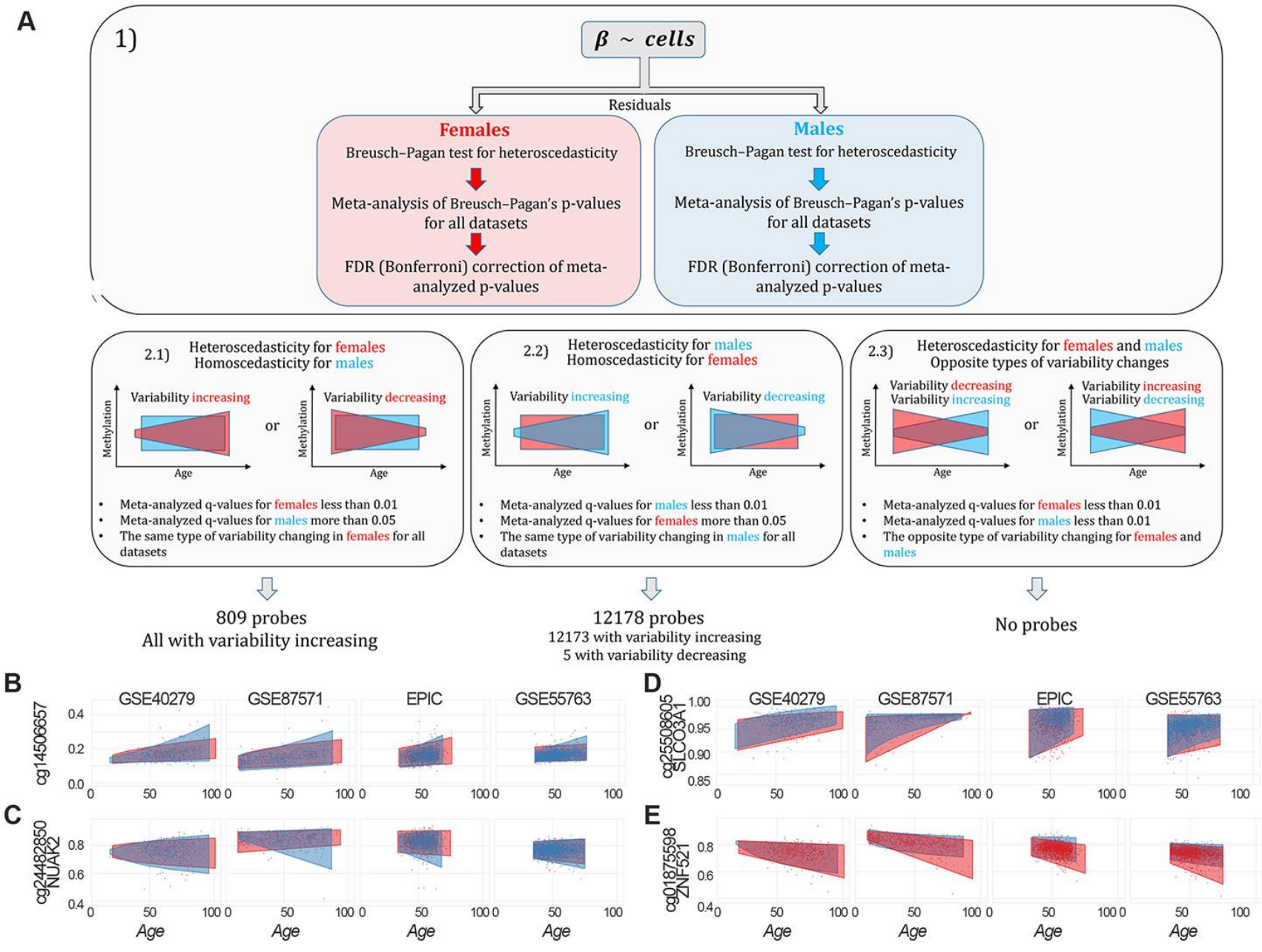

Figure 4. Identification of sex-specific age-associated variably methylated positions (saVMPs) (A) The approach used to identify saVMPs. (B-E) Some examples of saVMPs showing age-associated increase in variability in males (B, C), age-associated decrease in variability in males (D) or age-associated increase in variability in females (E). $x$ axis corresponds to age of subjects, $y$ axis to methylation levels. 
imprinted genes, but they did not find an enrichment in sex hormone-related genes [22]. Our list of saDMPs was enriched in $\mathrm{CpG}$ island shores but depleted in imprinted genes. We did not find any significant enrichment in sex-hormone related genes, although saDMPs included a higher proportion of this class of genes compared to snaDMPs. Finally, we searched for those probes displaying different DNA methylation trajectories in males and females during aging. We identified 8 probes having age-by-sex interaction, 2 of which were recently reported by McCartney et al. in an independent dataset [32]. In particular we found that FIGN gene included both probes from the saDMPs list and probes displaying an age-by-sex interaction.

It is difficult to speculate on the mechanisms that regulate saDMPs and on the functional consequences of this differential methylation in blood. By way of example, we considered the genes in which the 5 top ranker saDMPs map and we evaluated their tissuespecific gene expression profiles in the GTEx portal (https://www.gtexportal.org/home/). FIGN gene encodes for Fidgetin protein, an ATP-dependent microtubule severing enzyme that catalyses internal breaks in microtubules and is involved in different cellular processes, including cell division and neurogenesis [38]. The gene is poorly expressed in whole blood, while it is expressed at higher levels in arteries and female reproductive organs. DOC2A (Double C2 Domain Alpha) is mainly expressed in testis and brain, where it acts as a $\mathrm{Ca} 2+$ sensor and regulates asynchronous neurotransmitter release [39]. PEX10 (Peroxisomal Biogenesis Factor 10) encodes for a protein localized to the peroxisomal membrane and involved in the import of peroxisomal matrix proteins. The gene is highly expressed in testis, and a metaanalysis suggests that PEX10 polymorphisms are associated with male infertility, especially with nonobstructive azoospermia susceptibility [40]. PRR4 gene encodes for Proline-rich protein 4, a poorly characterized protein with very low expression in most human tissues, except for tear fluid [41]. It is worth of note that several of these genes are expressed in reproductive organs, and we cannot exclude that the observed epigenetic changes in whole blood are a proxy of what happens in these tissues. None of these genes was differentially expressed in PBMCs from nonagenarians with respect to young controls (GSE65219), or when males and females from the same cohort were compared [42]. Notwithstanding, using the "Ominer" tool in the Signaling Pathways Project [43] website (https://www.signalingpathways.org/ominer/ query.jsf), we found that all the 4 genes are regulated in cellular models (mainly epithelial cells) treated with bioactive small molecules that bind to estrogen and androgen receptors (Supplementary File 7). Future studies would clarify whether sex- and age-dependent methylation of these genes occurs also in tissues different from blood, whether this affects their expression and whether hormones directly regulate them across the lifespan.

\section{saDMPs in centenarians and Down syndrome persons.}

Another question is whether the saDMPs that we identified are modulated according to biological age. To this aim, we exploited two cohorts available in our lab, in which we measured whole blood DNA methylation by the targeted EpiTYPER assay: subjects affected by Down syndrome, as a model of premature/accelerated aging [34, 35], and centenarians, as a model of successful/decelerated aging [14]. The results are intriguing, as both models showed a peculiar sex specific alteration in FIGN and PRR4 epigenetic
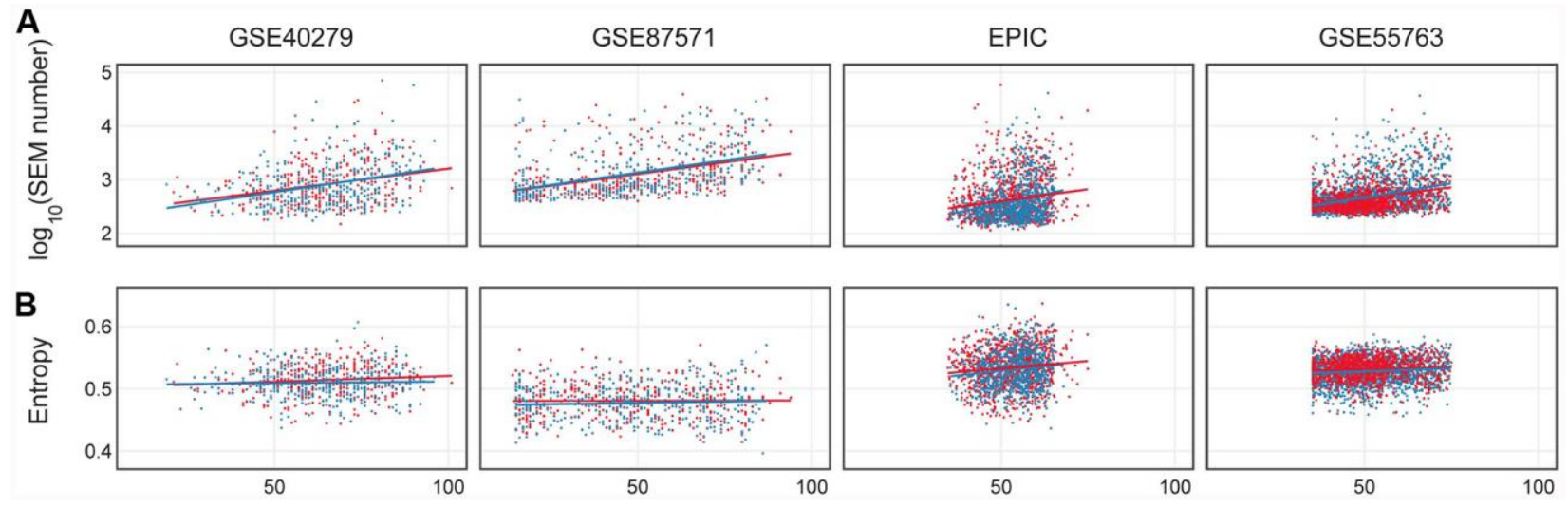

Figure 5. (A) Number of epimutations (log scale) in dependence on age in females (red) and males (blue). (B) Shannon entropy for 4 considered datasets: GSE40279, GSE87571, EPIC, GSE55763. 
patterns. In particular, a subset of centenarian males showed a "feminization" of FIGN methylation values, while females with Down syndrome showed a "masculinization" of PRR4 methylation values. No differences were found in the centenarians' offspring group, despite we and others previously showed an epigenetic age deceleration effect in these subjects [14]. Interestingly, the "feminization" of centenarians methylation profiles at FIGN locus is reminiscent of the gene expression shift towards female patterns observed after caloric restriction [44, 45]. Further studies should deepen these results and identify other changes in saDMPs that are associated with agerelated diseases or longevity.

\section{saVMPs in healthy subjects of different ages and populations.}

An increase in epigenetic variability has been reported during aging [6], in line with what observed for other molecular layers [46-48]. In the GSE87571 cohort we showed a global increase in DNA methylation variance during aging, and we further reported a trend towards higher variance in males compared to females at older ages. This result mimics what was observed for gene expression in the hippocampus of male and female mice at different ages [49], thus suggesting that the loss of epigenetic and transcriptional control that occurs during aging is more marked in males than in females. Accordingly, a more specific search for saVMPs showed that in males the number of probes displaying age-related changes in methylation variability is 15 times higher than in females and that the vast majority of these probes undergo an increase in age-related variability, as previously reported [6]. Interestingly, this list was significantly enriched in imprinted regions. An increase in variability at these loci can be related to the phenomenon of loss of imprinting, which has been largely reported in cancer and demonstrated to occur during aging [50-52].

\section{Epimutations and entropy}

While variable probes are defined at the level of population, epimutations are rare methylation changes that are specific for one or few individuals within a certain population. As such, variable probes and epimutations represent distinct aspects of epigenetic instability, that can be differently triggered during aging and that can differently affect aging trajectories. Accumulation of epimutations has been reported in cancer [53], and we and others demonstrated that the number of epimutations increases with age [5, 54]. Recently, Wang and colleagues showed that the number of epimutations in whole blood tends to be higher in females compared to males [54]. On the contrary, we failed to detect differences in the agerelated increase in epimutations between the two sexes. This discrepancy can be due to the different analytical approaches and/or to cohort-specific effects, as Wang et al investigated monozygotic and dizygotic twins longitudinally assessed. A recent paper assessed epimutations in 3 large cohorts and did not find significant differences between males and females [55]. Similarly, we did not find sex-related differences in age-related changes in Shannon entropy, another measure of epigenetic drift.

\section{Strengths and Limitations}

The main strengths of our work are: i) we compared the two sexes not only for age-associated hyper- or hypo-methylation changes, but also for other types of epigenetic remodeling (variability, epimutations, entropy) that, although less characterized, are likely to affect aging and be involved in the sex gap in longevity; ii) the analysis was performed in 4 distinct datasets including subjects recruited in different geographic area (United States, Sweden, Italy, United Kingdom) and belonging to different ethnic groups (European, Hispanic, Indian Asian). Population epigenetics is an emerging field. Some studies reported that epigenetic clock estimations can differ between ethnic groups, and that sex-dependent differences can be specific for some populations [26, 27]. Our meta-analysis of datasets deriving from different populations and ethnic groups allowed us to disentangle the effects of sex from those of potentially confounding factors, like genetic background and socio-cultural aspects related to gender definition; iii) beside healthy individuals representative of physiological aging, we evaluated also extreme phenotypes (persons with Down Syndrome, centenarians and their offspring) that provide a first descriptive insight on the possible contribution of sexspecific methylation in the sex gap in aging and longevity.

At the same time, our study has some limitations. The analyzed datasets differ in terms of size, age-range and data pre-processing procedures (in particular the GSE40279 dataset). It is therefore likely that our selection excluded additional $\mathrm{CpG}$ sites displaying a sex-specificity in their age-associated methylation trends, but not evident in all the datasets due to the above-mentioned differences between them. Furthermore, although some ethnic groups are included, many are missing, and the study of additional populations will be necessary to distinguish the effects of sex and gender in shaping age-related methylation changes. Finally, the first step of our pipeline (the identification of sDMPs) prevented us to 
include in the analysis sex chromosomes, similarly to [22]. The analysis of sex- and age-dependent DNA methylation on sex chromosomes poses peculiar technical difficulties, due to the different number of $\mathrm{X}$ chromosomes in females and males and to the $\mathrm{X}$ chromosome inactivation (XCI) process in females. By normalizing raw methylation data in males and females separately (an approach not applicable to GSE40279 and GSE55763 datasets included in our study, as raw data were not available in GEO), $\mathrm{Li}$ et al. recently reported a small number of $\mathrm{CpG}$ sites on $\mathrm{X}$ chromosome having sex-specific age-dependent changes in relation to XCI [56]. Similarly, Lund et al. evaluated the changes in $\mathrm{Y}$ chromosome methylation during aging [57]. Future studies based on alternative approaches, like allele-specific analysis of bisulfitesequencing data, will help in disentangling the dynamics of sex chromosomes methylation with aging, taking into account also possible age-dependent changes in XCI.

\section{CONCLUSIONS AND FUTURE PERSPECTIVES}

In conclusion, we provided a comprehensive description of sex-differences in DNA methylation changes with aging in whole blood. Our results suggest that a large fraction of $\mathrm{CpG}$ sites with sex-specific DNA methylation patterns are also modulated during aging, and that sex can affect some aspects of agerelated epigenetic remodeling, like an increase in variability in DNA methylation patterns. Future studies should investigate the tissue-specificity of these patterns and their relationship with gene expression differences between males and females, in particular for those probes that show age-by-sex interactions, to identify possible molecular triggers of sex gap in aging and longevity. Importantly, here we reported also a list of sex- but not age-associated probes, and we cannot exclude that also these sites can contribute to the dimorphism in aging phenotypes between males and females.

Our results pave the way for the development of a new generation of sex-specific epigenetic clocks that, compared to the "unisex" clocks currently available, are likely to be more informative of the peculiar trajectories that males and females experience during aging.

\section{MATERIALS AND METHODS}

\section{Datasets}

The Gene Expression Omnibus (GEO) Datasets repository [58] was interrogated using "GPL13534" (the accession code of the platform HumanMethylation450 BeadChip, Illumina) and "blood" as search terms, setting "tissue", "age", "gender" and "sex" as attributes and sorting the results by Number of Samples (High to Low). Only datasets including healthy subjects were considered. Based on these criteria, as to June 1st 2019 we selected the 3 datasets including the highest number of samples: GSE40279 [31], GSE87571 [59] and GSE55763 [60]. Furthermore, we analyzed a fourth dataset not uploaded in GEO that is part of the EPIC Italy study [61]. The total number of subjects included in each dataset, as well as the number of males and females, are reported in Supplementary Table 2. Supplementary Figure 7 reports, for each dataset, the number of males and females according to age.

For the EPIC dataset, raw data were normalized using an in-house software written for the R environment and extensively described in [62]. For the datasets downloaded from GEO, raw data (.idat files) were available only for GSE87571. We extracted .idat files using minfi Bioconductor package and normalized them using the preprocessFunnorm function implemented in the same package [63]. For the remaining datasets, the analyses were performed on pre-processed beta value matrixes available in GEO: according to authors' indications, the GSE55763 data were the result of a quantile normalization of intensity values, while GSE40279 beta values were not normalized but adjusted for internal controls by the Illumina's Genome Studio software.

Probes mapping on sex chromosomes and probes with internal SNPs, with non-unique mapping to the bisulfite-converted genome and with off-target hybridization according to [64] were excluded from each dataset, leaving 414505 probes for GSE40279, 414950 probes for GSE87571, 349534 probes for EPIC and 382458 probes for GSE55763. 327905 probes were common to the four datasets and were considered in the analyses described below.

In each dataset, blood cell proportions were estimated from methylation data using Horvath's calculator [65].

\section{Identification of age-associated probes having sex- specific DNA methylation patterns}

To identify $\mathrm{CpG}$ sites showing DNA methylation differences between the two sexes and age-associated changes in DNA methylation (sex- and age-associated differentially methylated positions, saDMPs), we proceeded as follows (Figure 1A): 1) We metaanalyzed the 4 datasets to identify probes with sexdependent DNA methylation patterns (sDMPs). As 
previously described [22], in each dataset we calculated the p-value of Pearson's correlation between sex and methylation beta-values, previously adjusted for age and blood cell proportions (CD8T cells, CD4T cells, NK cells, B cells and granulocytes, estimated as described above). METAL [66] was used to perform sample-size weighted meta-analysis on the 4 lists of Benjamini-Hochberg corrected p-values. 2) We metaanalyzed the 4 datasets to identify probes with agedependent DNA methylation patterns (aDMPs). In each dataset, we calculated the p-value of Pearson's correlation between age and methylation beta-values, previously adjusted for sex and blood cell proportions (CD8T cells, CD4T cells, NK cells, B cells and granulocytes, estimated as described above). As described above, METAL [66] was used to perform a sample-size weighted meta-analysis on the 4 lists of Benjamini-Hochberg p-values. 3) The meta-analyzed pvalues were corrected using Bonferroni procedure and a significance threshold of 0.01 was considered. Furthermore, only probes with a concordant trend in all the 4 datasets (for sDMPs: hypermethylated or hypomethylated in males respect to females in all the datasets; for aDMPs: hypermethylated of hypomethylated with age in all the datasets) were considered, returning a list of 38100 sDMPs and a list of 87581 aDMPs. 4) Finally, to identify saDMPs, we intersected the list of sDMPs and the list of aDMPs, resulting in a list of 16526 probes.

\section{Identification of probes having sex-specific trends in age-associated methylation variability}

To identify probes having sex-specific differences in age-dependent variability of methylation (sex-specific age-associated variably methylated positions, ssaVMPs) we proceeded as follows (Figure 3B): 1) We first regressed out the estimates of CD8T cells, CD4T cells, NK cells, B cells and granulocytes from beta values in each dataset; 2) To check for heteroscedasticity respect to age, we applied the Breusch-Pagan for males and females separately in each dataset [67, 68]. 3) Heteroscedasticity p-values were analyzed with sample-size weighted metaanalysis using METAL [66] for males and females separately. The meta-analyzed p-values were corrected using Bonferroni procedure and a significance threshold of 0.01 was considered. 4) We defined 3 possible scenarios of sex-specific differences in agedependent variability of methylation: a) Probes heteroscedastic in females and homoscedastic in males, that is probes with a meta-analyzed and Bonferronicorrected p-value less than 0.01 in females and higher than 0.05 in males. b) Probes homoscedastic in females and heteroscedastic in males, that is probes with a meta-analyzed and Bonferroni-corrected p-value less than 0.01 in males and higher than 0.05 in females. c) Probes heteroscedastic in both females and males, but with opposite directions of change in variability (variability increases in females and decreases in males or variability decreases in females and increases in males).

\section{Identification of epimutations and Shannon entropy analysis}

To identify epimutations (i.e., $\mathrm{CpG}$ probes for which one or few individuals show extremely different methylation levels compared to the rest of the cohort), for each probe we calculated the interquartile ranges of beta values; we then selected the probes having one or more subjects (epimutated subjects) having a beta value exceeding three times interquartile ranges $(\mathrm{Q} 1$ $(3 \times \mathrm{IQR})$ and $\mathrm{Q} 3+(3 \times \mathrm{IQR}))$, as reported in [5].

To calculate Shannon entropy, we applied the following procedure, according to [69]: 1) we obtained residuals by filtering out the dependence of beta-values on blood cells proportions; 2) we recalculated betavalues according to the formula:

$$
\beta_{i, j}^{a d j}=\text { residuals }_{i, j}+\operatorname{mean}\left(b_{i}\right)
$$

where mean $\left(\beta_{i}\right)$ is the average methylation level for $\mathrm{i}^{\text {th }}$ CpG site, $i$ is the index of CpG and $\mathrm{j}$ is the index of subject. Then, we calculated Shannon entropy using the following formula, as indicated in [31]:

$$
\begin{aligned}
& \text { Entropy }=1 / N \cdot \log (1 / 2) \sum_{i}\left[\beta_{i}^{a d j} \cdot \log \left(\beta_{i}^{a d j}\right)+\right. \\
& \left.\left(1-\beta_{i}^{a d j}\right) \cdot \log \left(1-\beta_{i}^{a d j}\right)\right]
\end{aligned}
$$

where $\beta_{i}^{a d j}$ is the recalculated methylation level for $\mathrm{i}^{\text {th }}$ CpG site and $N$ is the number of $\mathrm{CpG}$ sites.

For both epimutations and Shannon entropy, an ANCOVA model was calculated and adjusted for the interaction term between age and sex.

\section{Gene-targeted DNA methylation analysis}

The EpiTYPER assay (Agena) was used to measure DNA methylation of FIGN and PRR4 in whole blood from 560 subjects belonging to 4 groups: 419 healthy controls of different ages, 49 centenarians, 48 centenarians' offspring and 44 persons with Down Syndrome. Age range and sex distribution of the 4 cohorts are reported in Supplementary Table 3. All the subjects were recruited following the approval by the Ethical Committee of Sant'Orsola-Malpighi University Hospital (Bologna, Italy). 
Genomic DNA was extracted using the QIAamp 96 DNA Blood Kit (Qiagen) and 500 ng were bisulphite converted using EZ-96 DNA Methylation Kit (Zymo Research Corporation). Ten ng of bisulphite-converted DNA were amplified using the following bisulphitespecific primers, containing tag sequences for the EpiTYPER protocol: FIGN forward aggaagagagTTTTTTGAAAAGAGAGAAAGAAGGA ; FIGN reverse cagtaatacgactcactatagggagaaggct ATAAACAATCAAACCATCCAATTTCTA; $P R R 4$ forward aggaagagagTTTGTGTTTTGAGTTGAGTTT AGAG; PRR4 reverse cagtaatacgactcactatagggagaa ggctCCTAAAAATAAAACTTCTATCATCCA.

Primers for FIGN and PRR4 amplified chr2:164,589,883-164,590,418 and chr12:11, 001,978-11,002,636 (GRCh37/hg19 genome assembly) respectively.

\section{Enrichment analyses}

Enrichment of genomic regions, imprinted genes and sex hormone-related genes was calculated using Fisher exact test, as implemented in the fisher.test function in the stats $\mathrm{R}$ package. The lists of imprinted genomic regions [33] and of sex hormone-related genes used as background are reported in Supplementary File 8. Enrichment of GO annotations was calculated using the methylgometh function implemented in the methylGSA R package, using default settings [70].

\section{AUTHOR CONTRIBUTIONS}

IY, MGB, MI, and CF contributed to the conception and design of the study. IY, CP, CG, GF, AG, SP organized the datasets. IY, MGB, AK, MK, MV, CP, CG performed the statistical analysis. MGB, NG, FR, $\mathrm{MM}, \mathrm{CP}, \mathrm{CG}$ performed the validation experiments. IY, MGB, AK, PG, MI and CF wrote the manuscript. All authors contributed to manuscript revision, and read and approved the submitted version.

\section{CONFLICTS OF INTEREST}

The authors declare that they have no conflicts of interest.

\section{FUNDING}

We acknowledge the support of the Ministry of Science and Higher Education agreement No. 075-152019-871. Epimutations and entropy analysis were performed under the Ministry of Science and Higher Education agreement No. 075-15-2020-808. This work was supported by the European Union (EU)'S H2020 Project "PROPAG-AGEING" (grant agreement 634821) and by the EU JPND "ADAGE.

\section{REFERENCES}

1. Bacalini MG, D'Aquila P, Marasco E, Nardini C, Montesanto A, Franceschi C, Passarino G, Garagnani P, Bellizzi D. The methylation of nuclear and mitochondrial DNA in ageing phenotypes and longevity. Mech Ageing Dev. 2017; 165:156-61.

https://doi.org/10.1016/i.mad.2017.01.006

PMID:28115210

2. Ciccarone F, Tagliatesta S, Caiafa P, Zampieri M. DNA methylation dynamics in aging: how far are we from understanding the mechanisms? Mech Ageing Dev. 2018; 174:3-17.

https://doi.org/10.1016/j.mad.2017.12.002

PMID:29268958

3. Ashapkin VV, Kutueva LI, Vanyushin BF. Epigenetic clock: just a convenient marker or an active driver of aging? Adv Exp Med Biol. 2019; 1178:175-206.

https://doi.org/10.1007/978-3-030-25650-0 10 PMID:31493228

4. Feil R, Fraga MF. Epigenetics and the environment: emerging patterns and implications. Nat Rev Genet. 2012; 13:97-109.

https://doi.org/10.1038/nrg3142 PMID:22215131

5. Gentilini D, Garagnani P, Pisoni S, Bacalini MG, Calzari L, Mari D, Vitale G, Franceschi C, Di Blasio AM. Stochastic epigenetic mutations (DNA methylation) increase exponentially in human aging and correlate with $X$ chromosome inactivation skewing in females. Aging (Albany NY). 2015; 7:568-78.

https://doi.org/10.18632/aging.100792 PMID:26342808

6. Slieker $\mathrm{RC}$, van Iterson $\mathrm{M}$, Luijk $\mathrm{R}$, Beekman $\mathrm{M}$, Zhernakova DV, Moed MH, Mei H, van Galen M, Deelen $P$, Bonder MJ, Zhernakova A, Uitterlinden AG, Tigchelaar $\mathrm{EF}$, et al, and BIOS consortium. Age-related accrual of methylomic variability is linked to fundamental ageing mechanisms. Genome Biol. 2016; 17:191.

https://doi.org/10.1186/s13059-016-1053-6 PMID:27654999

7. Jones MJ, Goodman SJ, Kobor MS. DNA methylation and healthy human aging. Aging Cell. 2015; 14:924-32. https://doi.org/10.1111/acel.12349 PMID:25913071

8. Unnikrishnan A, Freeman WM, Jackson J, Wren JD, Porter $\mathrm{H}$, Richardson A. The role of DNA methylation in epigenetics of aging. Pharmacol Ther. 2019; 195:172-85.

https://doi.org/10.1016/i.pharmthera.2018.11.001 PMID:30419258

9. Zhu T, Zheng SC, Paul DS, Horvath S, Teschendorff AE. Cell and tissue type independent age-associated DNA methylation changes are not rare but common. Aging (Albany NY). 2018; 10:3541-57. 
https://doi.org/10.18632/aging.101666

PMID:30482885

10. Slieker RC, Relton CL, Gaunt TR, Slagboom PE, Heijmans BT. Age-related DNA methylation changes are tissue-specific with ELOVL2 promoter methylation as exception. Epigenetics Chromatin. 2018; 11:25. https://doi.org/10.1186/s13072-018-0191-3 PMID:29848354

11. Bell CG, Lowe R, Adams PD, Baccarelli AA, Beck S, Bell JT, Christensen BC, Gladyshev VN, Heijmans BT, Horvath S, Ideker T, Issa JJ, Kelsey KT, et al. DNA methylation aging clocks: challenges and recommendations. Genome Biol. 2019; 20:249. https://doi.org/10.1186/s13059-019-1824-y PMID:31767039

12. Fransquet PD, Wrigglesworth J, Woods RL, Ernst ME, Ryan J. The epigenetic clock as a predictor of disease and mortality risk: a systematic review and metaanalysis. Clin Epigenetics. 2019; 11:62. https://doi.org/10.1186/s13148-019-0656-7 PMID:30975202

13. Horvath S, Raj K. DNA methylation-based biomarkers and the epigenetic clock theory of ageing. Nat Rev Genet. 2018; 19:371-84. https://doi.org/10.1038/s41576-018-0004-3 PMID:29643443

14. Horvath S, Pirazzini C, Bacalini MG, Gentilini D, Di Blasio AM, Delledonne M, Mari D, Arosio B, Monti D, Passarino G, De Rango F, D'Aquila P, Giuliani C, et al. Decreased epigenetic age of PBMCs from italian semisupercentenarians and their offspring. Aging (Albany NY). 2015; 7:1159-70.

https://doi.org/10.18632/aging.100861 PMID:26678252

15. Fahy GM, Brooke RT, Watson JP, Good Z, Vasanawala SS, Maecker H, Leipold MD, Lin DT, Kobor MS, Horvath S. Reversal of epigenetic aging and immunosenescent trends in humans. Aging Cell. 2019; 18:e13028. https://doi.org/10.1111/acel.13028 PMID:31496122

16. World Health Organization. Global Health Estimates: Life expectancy and leading causes of death and disability.

http://www.who.int/gho/mortality burden disease/ life tables/situation trends text/en/

17. Austad SN, Fischer KE. Sex differences in lifespan. Cell Metab. 2016; 23:1022-33.

https://doi.org/10.1016/i.cmet.2016.05.019 PMID:27304504

18. Ostan R, Monti D, Gueresi P, Bussolotto M, Franceschi C, Baggio G. Gender, aging and longevity in humans: an update of an intriguing/neglected scenario paving the way to a gender-specific medicine. Clin Sci (Lond). 2016; 130:1711-25.

https://doi.org/10.1042/CS20160004 PMID:27555614

19. Giuliani C, Garagnani P, Franceschi C. Genetics of human longevity within an eco-evolutionary naturenurture framework. Circ Res. 2018; 123:745-72. https://doi.org/10.1161/CIRCRESAHA.118.312562 PMID:30355083

20. Zeng Y, Nie C, Min J, Chen H, Liu X, Ye R, Chen Z, Bai C, Xie E, Yin Z, Lv Y, Lu J, Li J, et al. Sex differences in genetic associations with longevity. JAMA Netw Open. 2018; 1:e181670.

https://doi.org/10.1001/jamanetworkopen.2018.1670 PMID:30294719

21. Inoshita $M$, Numata $S$, Tajima $A$, Kinoshita $M$, Umehara $\mathrm{H}$, Yamamori $\mathrm{H}$, Hashimoto R, Imoto I, Ohmori T. Sex differences of leukocytes DNA methylation adjusted for estimated cellular proportions. Biol Sex Differ. 2015; 6:11.

https://doi.org/10.1186/s13293-015-0029-7 PMID:26113971

22. Singmann $P$, Shem-Tov D, Wahl S, Grallert H, Fiorito G, Shin SY, Schramm K, Wolf P, Kunze S, Baran Y, Guarrera $S$, Vineis $P$, Krogh $V$, et al. Characterization of wholegenome autosomal differences of DNA methylation between men and women. Epigenetics Chromatin. 2015; 8:43.

https://doi.org/10.1186/s13072-015-0035-3 PMID:26500701

23. Yousefi P, Huen K, Davé V, Barcellos L, Eskenazi B, Holland N. Sex differences in DNA methylation assessed by $450 \mathrm{~K}$ BeadChip in newborns. BMC Genomics. 2015; 16:911. https://doi.org/10.1186/s12864-015-2034-y PMID:26553366

24. Gershoni M, Pietrokovski S. The landscape of sexdifferential transcriptome and its consequent selection in human adults. BMC Biol. 2017; 15:7. https://doi.org/10.1186/s12915-017-0352-z PMID:28173793

25. Lehallier B, Gate D, Schaum N, Nanasi T, Lee SE, Yousef $H$, Moran Losada P, Berdnik D, Keller A, Verghese J, Sathyan S, Franceschi C, Milman S, et al. Undulating changes in human plasma proteome profiles across the lifespan. Nat Med. 2019; 25:1843-50. https://doi.org/10.1038/s41591-019-0673-2 PMID:31806903

26. Horvath S, Gurven M, Levine ME, Trumble BC, Kaplan $H$, Allayee $H$, Ritz BR, Chen B, Lu AT, Rickabaugh TM, Jamieson BD, Sun D, Li S, et al. An epigenetic clock analysis of race/ethnicity, sex, and coronary heart disease. Genome Biol. 2016; 17:171. 
https://doi.org/10.1186/s13059-016-1030-0 PMID:27511193

27. Tajuddin SM, Hernandez DG, Chen BH, Noren Hooten N, Mode NA, Nalls MA, Singleton AB, Ejiogu N, Chitrala $K N$, Zonderman $A B$, Evans MK. Novel age-associated DNA methylation changes and epigenetic age acceleration in middle-aged African Americans and whites. Clin Epigenetics. 2019; 11:119.

https://doi.org/10.1186/s13148-019-0722-1

PMID:31426852

28. Li X, Ploner A, Wang Y, Magnusson PK, Reynolds C, Finkel D, Pedersen NL, Jylhävä J, Hägg S. Longitudinal trajectories, correlations and mortality associations of nine biological ages across 20-years follow-up. Elife. 2020; 9:e51507.

https://doi.org/10.7554/eLife.51507

PMID:32041686

29. Masser DR, Hadad N, Porter HL, Mangold CA, Unnikrishnan A, Ford MM, Giles CB, Georgescu C, Dozmorov MG, Wren JD, Richardson A, Stanford DR, Freeman WM. Sexually divergent DNA methylation patterns with hippocampal aging. Aging Cell. 2017; 16:1342-52.

https://doi.org/10.1111/acel.12681

PMID:28948711

30. Horvath S. DNA methylation age of human tissues and cell types. Genome Biol. 2013; 14:R115. https://doi.org/10.1186/gb-2013-14-10-r115 PMID:24138928

31. Hannum G, Guinney J, Zhao L, Zhang L, Hughes G, Sadda S, Klotzle B, Bibikova M, Fan JB, Gao Y, Deconde R, Chen M, Rajapakse I, et al. Genome-wide methylation profiles reveal quantitative views of human aging rates. Mol Cell. 2013; 49:359-67. https://doi.org/10.1016/i.molcel.2012.10.016 PMID:23177740

32. McCartney DL, Zhang F, Hillary RF, Zhang Q, Stevenson AJ, Walker RM, Bermingham ML, Boutin T, Morris SW, Campbell A, Murray AD, Whalley HC, Porteous DJ, et al. An epigenome-wide association study of sex-specific chronological ageing. Genome Med. 2019; 12:1. https://doi.org/10.1186/s13073-019-0693-z PMID:31892350

33. Court F, Tayama C, Romanelli V, Martin-Trujillo A, Iglesias-Platas I, Okamura K, Sugahara N, Simón C, Moore H, Harness JV, Keirstead H, Sanchez-Mut JV, Kaneki E, et al. Genome-wide parent-of-origin DNA methylation analysis reveals the intricacies of human imprinting and suggests a germline methylationindependent mechanism of establishment. Genome Res. 2014; 24:554-69. https://doi.org/10.1101/gr.164913.113
PMID:24402520

34. Franceschi C, Garagnani P, Gensous N, Bacalini MG, Conte $\mathrm{M}$, Salvioli S. Accelerated bio-cognitive aging in down syndrome: state of the art and possible deceleration strategies. Aging Cell. 2019; 18:e12903.

https://doi.org/10.1111/acel.12903

PMID:30768754

35. Horvath S, Garagnani P, Bacalini MG, Pirazzini C, Salvioli S, Gentilini D, Di Blasio AM, Giuliani C, Tung S, Vinters HV, Franceschi C. Accelerated epigenetic aging in down syndrome. Aging Cell. 2015; 14:491-95.

https://doi.org/10.1111/acel.12325

PMID:25678027

36. Yang $Y$, Kozloski M. Sex differences in age trajectories of physiological dysregulation: inflammation, metabolic syndrome, and allostatic load. J Gerontol A Biol Sci Med Sci. 2011; 66:493-500.

https://doi.org/10.1093/gerona/glr003 PMID:21350248

37. Scheinost D, Finn ES, Tokoglu F, Shen X, Papademetris $X$, Hampson M, Constable RT. Sex differences in normal age trajectories of functional brain networks. Hum Brain Mapp. 2015; 36:1524-35.

https://doi.org/10.1002/hbm.22720

PMID:25523617

38. McNally FJ, Roll-Mecak A. Microtubule-severing enzymes: from cellular functions to molecular mechanism. J Cell Biol. 2018; 217:4057-69.

https://doi.org/10.1083/jcb.201612104

PMID:30373906

39. Yao J, Gaffaney JD, Kwon SE, Chapman ER. Doc2 is a $\mathrm{Ca} 2+$ sensor required for asynchronous neurotransmitter release. Cell. 2011; 147:666-77.

https://doi.org/10.1016/i.cell.2011.09.046

PMID:22036572

40. Gu X, Li H, Chen X, Zhang X, Mei F, Jia M, Xiong C. PEX10, SIRPA-SIRPG, and SOX5 gene polymorphisms are strongly associated with nonobstructive azoospermia susceptibility. J Assist Reprod Genet. 2019; 36:759-68.

https://doi.org/10.1007/s10815-019-01417-w PMID:30863997

41. Perumal N, Funke S, Pfeiffer N, Grus FH. Characterization of lacrimal proline-rich protein 4 (PRR4) in human tear proteome. Proteomics. 2014; 14:1698-709.

https://doi.org/10.1002/pmic.201300039 PMID:24782455

42. Marttila $S$, Jylhävä J, Nevalainen $T$, Nykter $M$, Jylhä $M$, Hervonen A, Tserel L, Peterson P, Hurme $M$. Transcriptional analysis reveals gender-specific 
changes in the aging of the human immune system. PLoS One. 2013; 8:e66229.

https://doi.org/10.1371/journal.pone.0066229

PMID:23776639

43. Ochsner SA, Abraham D, Martin K, Ding W, McOwiti A, Kankanamge W, Wang Z, Andreano K, Hamilton RA, Chen Y, Hamilton A, Gantner ML, Dehart M, et al. The signaling pathways project, an integrated 'omics knowledgebase for mammalian cellular signaling pathways. Sci Data. 2019; 6:252.

https://doi.org/10.1038/s41597-019-0193-4

PMID:31672983

44. Estep PW 3rd, Warner JB, Bulyk ML. Short-term calorie restriction in male mice feminizes gene expression and alters key regulators of conserved aging regulatory pathways. PLoS One. 2009; 4:e5242.

https://doi.org/10.1371/journal.pone.0005242 PMID:19370158

45. Tyshkovskiy A, Bozaykut $P$, Borodinova AA, Gerashchenko MV, Ables GP, Garratt M, Khaitovich P, Clish CB, Miller RA, Gladyshev VN. Identification and application of gene expression signatures associated with lifespan extension. Cell Metab. 2019; 30:57393.e8.

https://doi.org/10.1016/i.cmet.2019.06.018 PMID:31353263

46. Mahmoudi S, Mancini E, Xu L, Moore A, Jahanbani F, Hebestreit K, Srinivasan R, Li X, Devarajan K, Prélot L, Ang $C E$, Shibuya $Y$, Benayoun BA, et al. Heterogeneity in old fibroblasts is linked to variability in reprogramming and wound healing. Nature. 2019; 574:553-58.

https://doi.org/10.1038/s41586-019-1658-5 PMID:31645721

47. Viñuela A, Brown AA, Buil A, Tsai PC, Davies MN, Bell JT, Dermitzakis ET, Spector TD, Small KS. Agedependent changes in mean and variance of gene expression across tissues in a twin cohort. Hum Mol Genet. 2018; 27:732-41.

https://doi.org/10.1093/hmg/ddx424 PMID:29228364

48. Hernando-Herraez I, Evano B, Stubbs T, Commere PH, Jan Bonder M, Clark S, Andrews S, Tajbakhsh S, Reik W. Ageing affects DNA methylation drift and transcriptional cell-to-cell variability in mouse muscle stem cells. Nat Commun. 2019; 10:4361. https://doi.org/10.1038/s41467-019-12293-4 PMID:31554804

49. Mangold CA, Wronowski B, Du M, Masser DR, Hadad N, Bixler GV, Brucklacher RM, Ford MM, Sonntag WE, Freeman WM. Sexually divergent induction of microglial-associated neuroinflammation with hippocampal aging. J Neuroinflammation. 2017; $14: 141$. https://doi.org/10.1186/s12974-017-0920-8 PMID:28732515

50. Bennett-Baker PE, Wilkowski J, Burke DT. Ageassociated activation of epigenetically repressed genes in the mouse. Genetics. 2003; 165:2055-62. PMID: 14704185

51. Fu VX, Dobosy JR, Desotelle JA, Almassi N, Ewald JA, Srinivasan $R$, Berres $M$, Svaren J, Weindruch $R$, Jarrard DF. Aging and cancer-related loss of insulin-like growth factor 2 imprinting in the mouse and human prostate. Cancer Res. 2008; 68:6797-802.

https://doi.org/10.1158/0008-5472.CAN-08-1714 PMID:18701505

52. Pirazzini C, Giuliani C, Bacalini MG, Boattini A, Capri M, Fontanesi E, Marasco E, Mantovani V, Pierini M, Pini E, Luiselli D, Franceschi C, Garagnani P. Space/population and time/age in DNA methylation variability in humans: a study on IGF2/H19 locus in different italian populations and in mono- and di-zygotic twins of different age. Aging (Albany NY). 2012; 4:509-20.

https://doi.org/10.18632/aging.100476 PMID:22879348

53. Teschendorff $A E$, Gao $Y$, Jones $A$, Ruebner $M$, Beckmann MW, Wachter DL, Fasching PA, Widschwendter M. DNA methylation outliers in normal breast tissue identify field defects that are enriched in cancer. Nat Commun. 2016; 7:10478.

https://doi.org/10.1038/ncomms10478 PMID:26823093

54. Wang Y, Karlsson R, Jylhävä J, Hedman ÅK, Almqvist C, Karlsson IK, Pedersen NL, Almgren M, Hägg $S$. Comprehensive longitudinal study of epigenetic mutations in aging. Clin Epigenetics. 2019; 11:187.

https://doi.org/10.1186/s13148-019-0788-9

PMID:31818313

55. Seeboth A, McCartney DL, Wang Y, Hillary RF, Stevenson AJ, Walker RM, Campbell A, Evans KL, Mclntosh AM, Hägg S, Deary IJ, Marioni RE. DNA methylation outlier burden, health, and ageing in generation Scotland and the lothian birth cohorts of 1921 and 1936. Clin Epigenetics. 2020; 12:49.

https://doi.org/10.1186/s13148-020-00838-0 PMID:32216821

56. Li S, Lund JB, Christensen K, Baumbach J, Mengel-From J, Kruse T, Li W, Mohammadnejad A, Pattie A, Marioni RE, Deary IJ, Tan Q. Exploratory analysis of age and sex dependent DNA methylation patterns on the $\mathrm{X}$ chromosome in whole blood samples. Genome Med. 2020; 12:39. https://doi.org/10.1186/s13073-020-00736-3 PMID:32345361

57. Lund JB, Li S, Christensen K, Mengel-From J, Soerensen 
M, Marioni RE, Starr J, Pattie A, Deary IJ, Baumbach J, Tan Q. Age-dependent DNA methylation patterns on the $\mathrm{Y}$ chromosome in elderly males. Aging Cell. 2020; 19:e12907.

https://doi.org/10.1111/acel.12907

PMID:30793472

58. Barrett T, Wilhite SE, Ledoux P, Evangelista C, Kim IF, Tomashevsky M, Marshall KA, Phillippy KH, Sherman PM, Holko M, Yefanov A, Lee $H$, Zhang $N$, et al. NCBI GEO: archive for functional genomics data setsupdate. Nucleic Acids Res. 2013; 41:D991-95.

https://doi.org/10.1093/nar/gks1193

PMID:23193258

59. Johansson A, Enroth S, Gyllensten U. Continuous aging of the human DNA methylome throughout the human lifespan. PLoS One. 2013; 8:e67378.

https://doi.org/10.1371/journal.pone.0067378 PMID:23826282

60. Lehne B, Drong AW, Loh M, Zhang W, Scott WR, Tan ST, Afzal U, Scott J, Jarvelin MR, Elliott P, McCarthy MI, Kooner JS, Chambers JC. A coherent approach for analysis of the illumina HumanMethylation450 BeadChip improves data quality and performance in epigenome-wide association studies. Genome Biol. 2015; $16: 37$.

https://doi.org/10.1186/s13059-015-0600-x

PMID:25853392

61. Palli $D$, Berrino $F$, Vineis $P$, Tumino $R$, Panico $S$, Masala G, Saieva C, Salvini S, Ceroti M, Pala V, Sieri S, Frasca G, Giurdanella MC, et al, and EPIC-Italy. A molecular epidemiology project on diet and cancer: the EPIC-italy prospective study. Design and baseline characteristics of participants. Tumori. 2003; 89:586-93.

PMID:14870823

62. Campanella G, Polidoro S, Di Gaetano C, Fiorito G, Guarrera S, Krogh V, Palli D, Panico S, Sacerdote C, Tumino R, Elliott P, Matullo G, Chadeau-Hyam M, Vineis $P$. Epigenetic signatures of internal migration in Italy. Int J Epidemiol. 2015; 44:1442-49.

https://doi.org/10.1093/ije/dyu198 PMID:25324151

63. Aryee MJ, Jaffe AE, Corrada-Bravo H, Ladd-Acosta C, Feinberg AP, Hansen KD, Irizarry RA. Minfi: a flexible and comprehensive bioconductor package for the analysis of infinium DNA methylation microarrays. Bioinformatics. 2014; 30:1363-69.

https://doi.org/10.1093/bioinformatics/btu049

PMID:24478339
64. Zhou W, Laird PW, Shen H. Comprehensive characterization, annotation and innovative use of infinium DNA methylation BeadChip probes. Nucleic Acids Res. 2017; 45:e22.

https://doi.org/10.1093/nar/gkw967

PMID:27924034

65. Horvath S. DNA Methylation Age Calculator. https://dnamage.genetics.ucla.edu/home

66. Willer CJ, Li Y, Abecasis GR. METAL: fast and efficient meta-analysis of genomewide association scans. Bioinformatics. 2010; 26:2190-91. https://doi.org/10.1093/bioinformatics/btq340 PMID:20616382

67. Breusch TS, Pagan AR. A Simple Test for Heteroscedasticity and Random Coefficient Variation. Econometrica. 1979; 47:1287-94. https://doi.org/10.2307/1911963

68. Cook RD, Weisberg S. Diagnostics for Heteroscedasticity in Regression. Biometrika. 1983; 70:1-10. https://doi.org/10.2307/1911963

69. Jones MJ, Islam SA, Edgar RD, Kobor MS. Adjusting for cell type composition in DNA methylation data using a regression-based approach. Methods Mol Biol. 2017; 1589:99-106. 10.2307/2335938 https://doi.org/10.1007/7651 2015262 PMID:26126446

70. Ren X, Kuan PF. methylGSA: a bioconductor package and shiny app for DNA methylation data length bias adjustment in gene set testing. Bioinformatics. 2019; 35:1958-59. https://doi.org/10.1093/bioinformatics/bty892 PMID:30346483 


\section{SUPPLEMENTARY MATERIALS}

\section{Supplementary Figures}
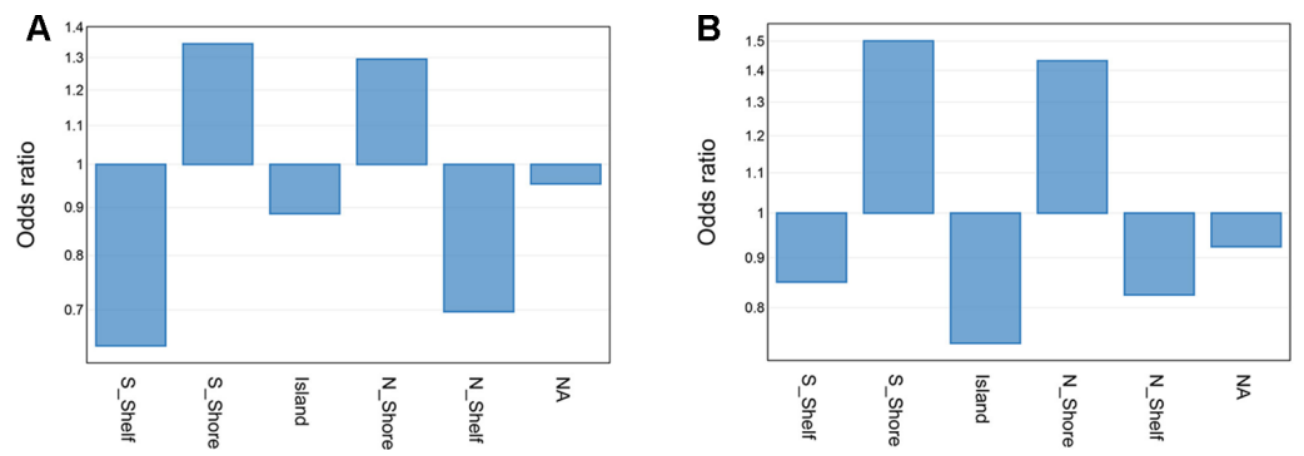

Supplementary Figure 1. Enrichment (odds ratio) of genomic localizations for saDMPs (A) and snaDMPs (B). 

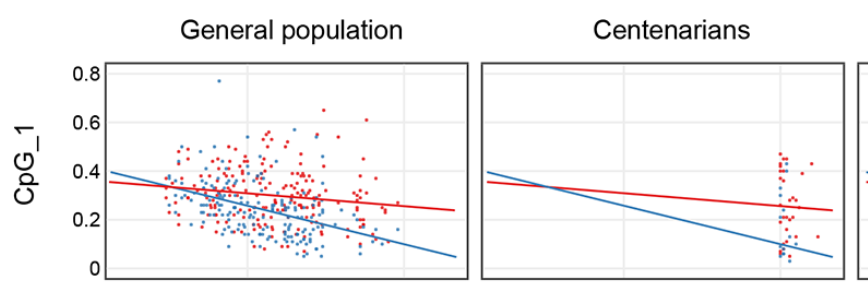

Centenarians' offspring

Persons with Down Syndrome
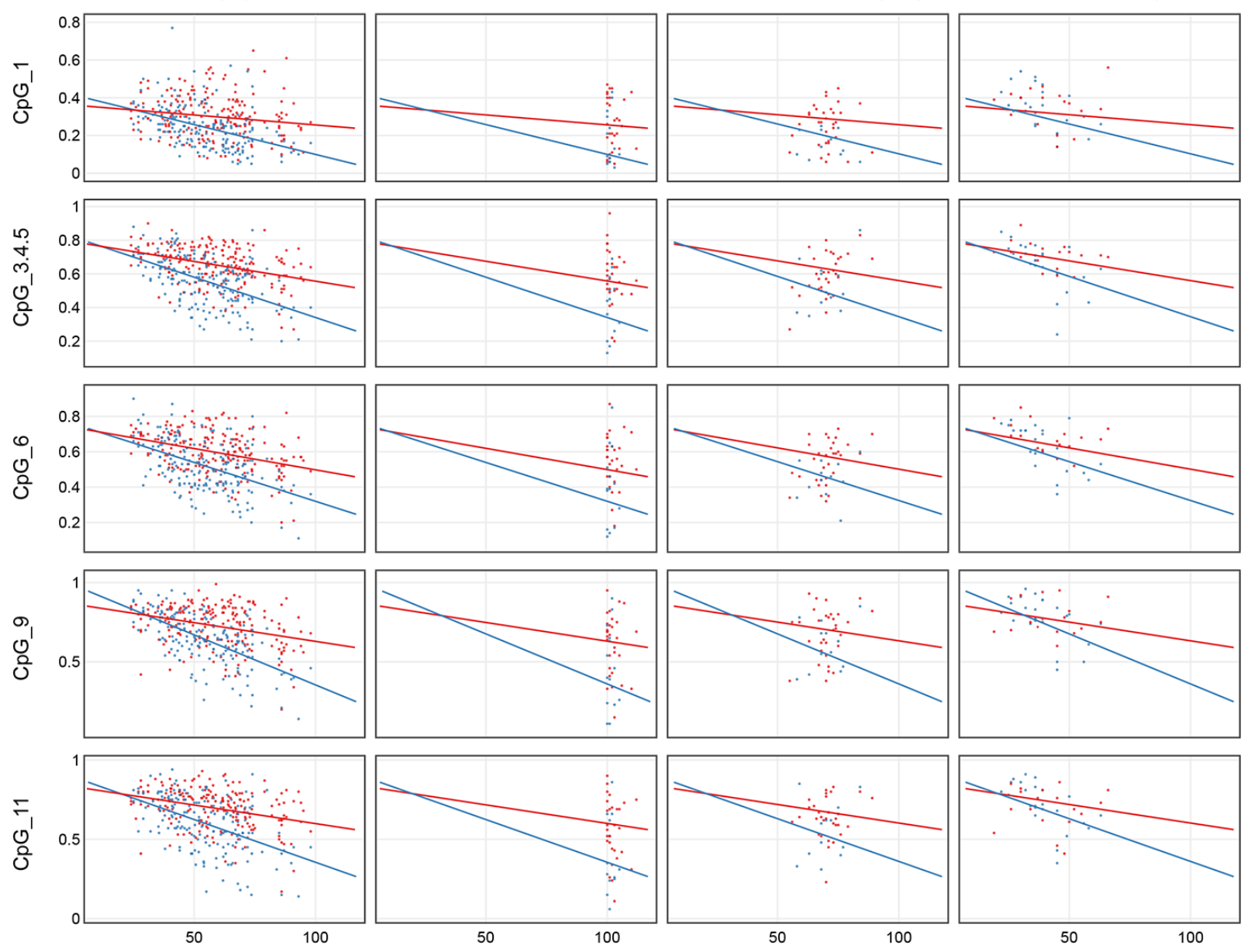

Supplementary Figure 2. Validation of FIGN locus by EpiTYPER. For each of the CpG units returned by the EpiTYPER assay, DNA methylation in controls (general population), centenarians, centenarian's offspring and persons with Down syndrome is reported vs the age of the subjects. Males are in blue, females are in red. Linear regression between DNA methylation and age was calculated separately for males and females in control subjects and was reported in each plot. 

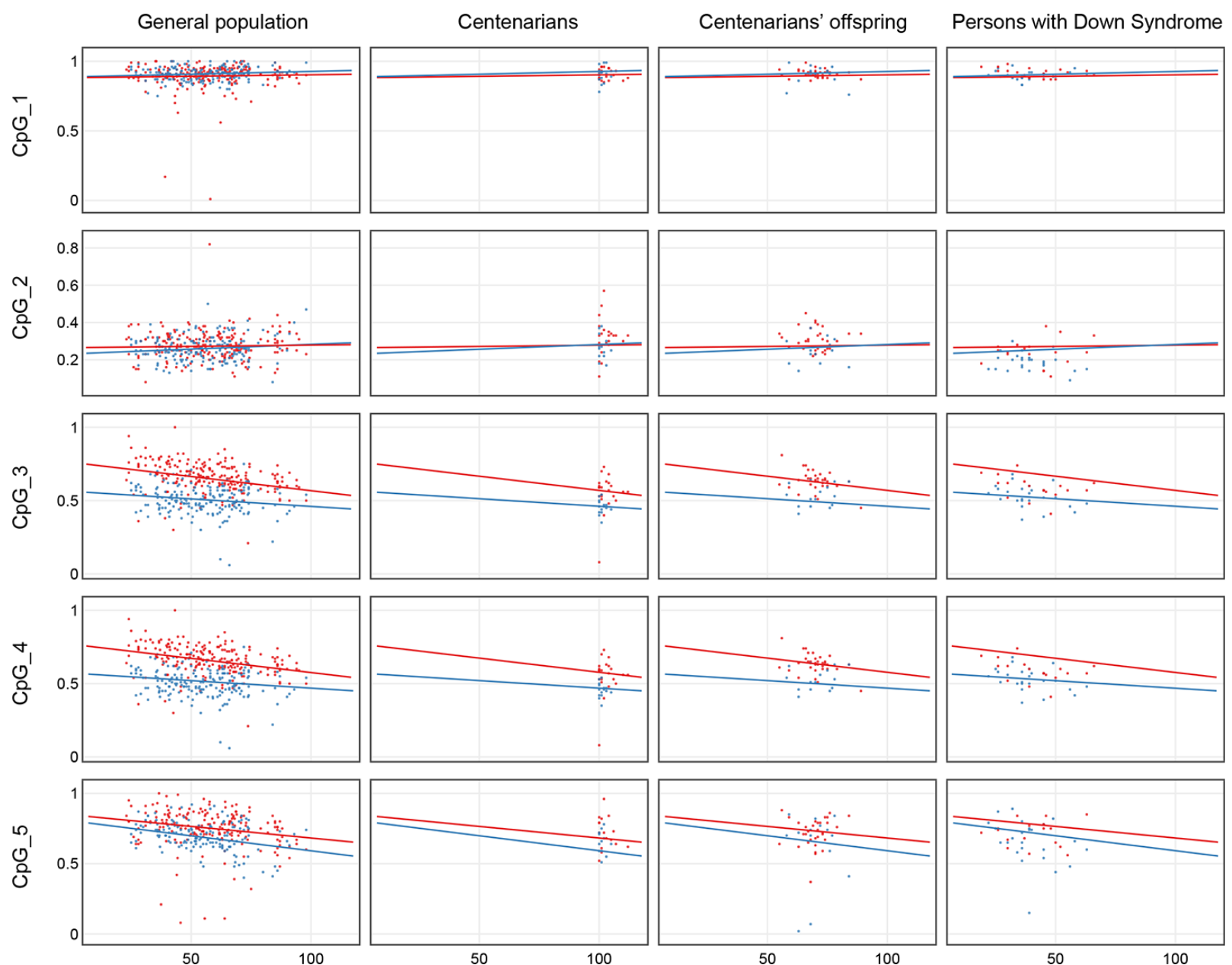

Supplementary Figure 3. Validation of PRR4 locus by EpiTYPER. For each of the 5 CpG units returned by the EpiTYPER assay, DNA methylation in controls (general population), centenarians, centenarian's offspring and persons with Down syndrome is reported vs the age of the subjects. Males are in blue, females are in red. Linear regression between DNA methylation and age was calculated separately for males and females in control subjects and was reported in each plot. 

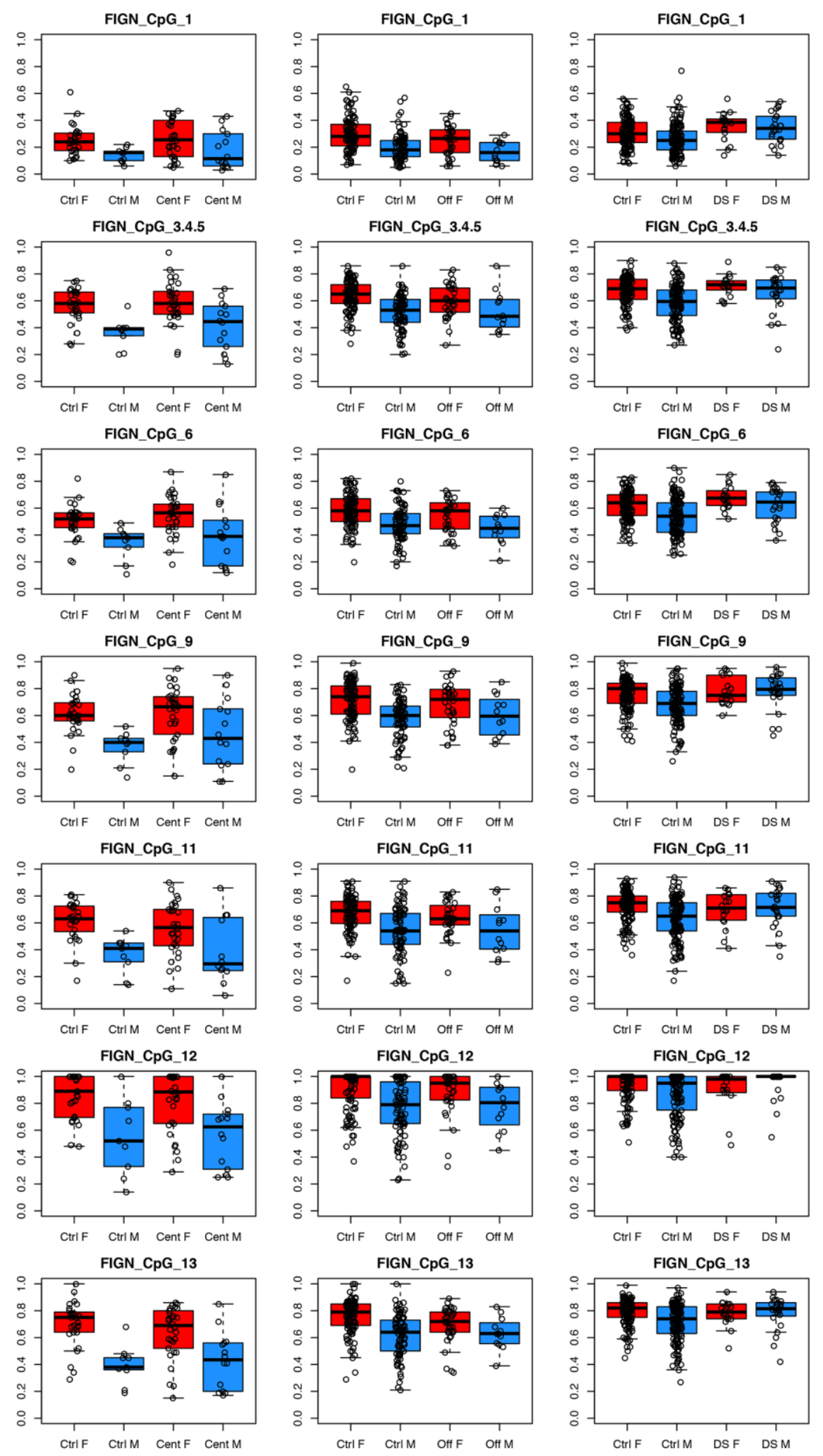

Supplementary Figure 4. Boxplots of DNA methylation for each CpG unit in FIGN amplicon in centenarians, centenarians' offspring and Down syndrome cohorts. Left panels: for each CpG unit in FIGN locus, boxplots of DNA methylation in male and female centenarians, compared to male and female controls $(>80,<100$ years). Middle panels: for each CpG unit in FIGN locus, boxplots of DNA methylation in male and females centenarians' offspring, compared to age-matched male and female controls $(>54,<90$ years). Right panels: for each CpG unit in FIGN locus, boxplots of DNA methylation in male and female Down syndrome persons, compared to age-matched male and female controls ( $>18,<67$ years). 
PRR4_CpG_1
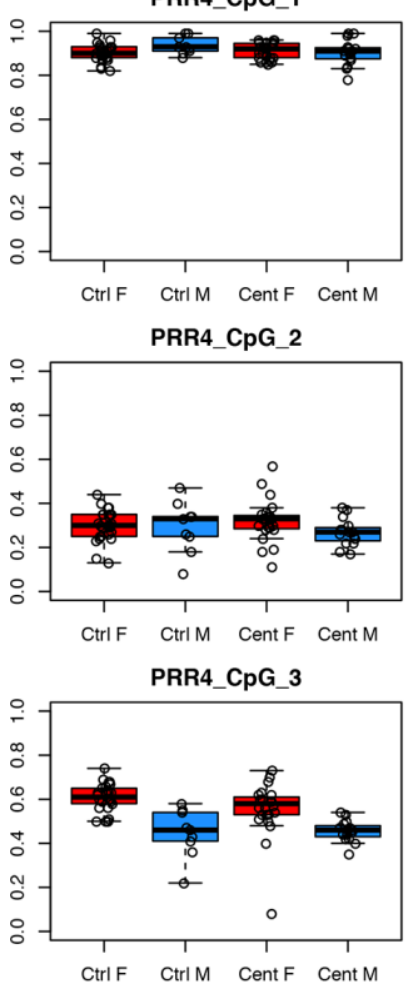

PRR4_CpG_4
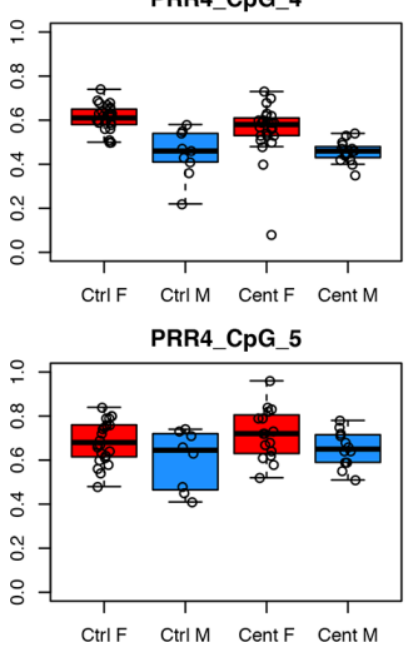

PRR4_CpG_1
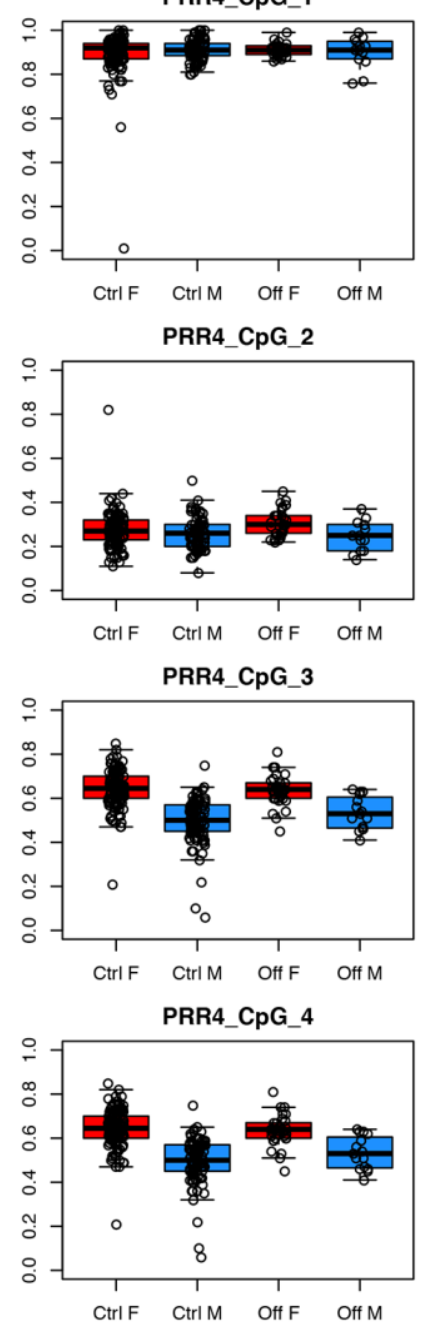

PRR4_CpG_5

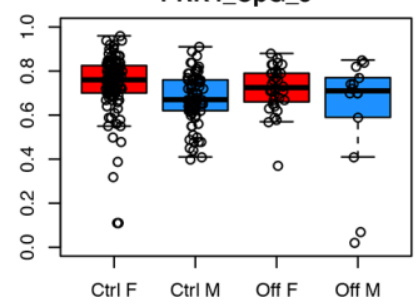

PRR4_CpG_1

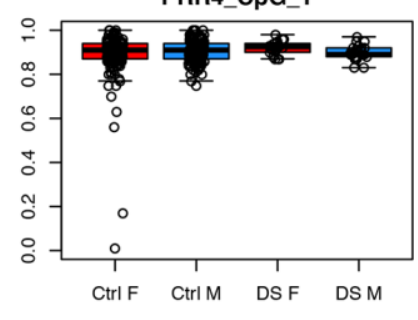

PRR4_CpG_2

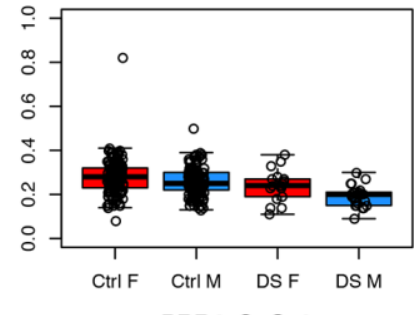

PRR4_CpG_3

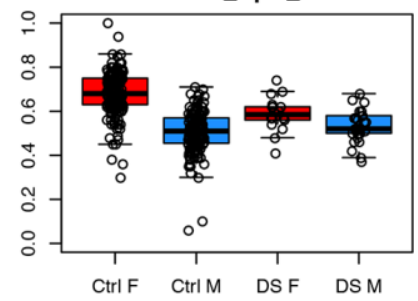

PRR4_CpG_4

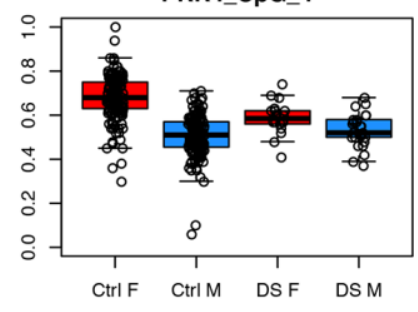

PRR4_CpG_5

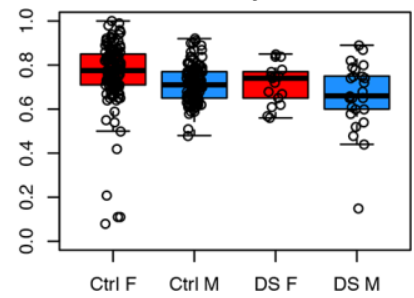

Supplementary Figure 5. Boxplots of DNA methylation for each CpG unit in PRR4 amplicon in centenarians, centenarians' offspring and Down syndrome cohorts. Left panels: for each CpG unit in PRR4 locus, boxplots of DNA methylation in male and female centenarians, compared to male and female controls ( $>80,<100$ years). Middle panels: for each CpG unit in PRR4 locus, boxplots of DNA methylation in male and females centenarians' offspring, compared to age-matched male and female controls ( $>54,<90$ years). Right panels: for each CpG unit in PRR4 locus, boxplots of DNA methylation in male and female Down syndrome persons, compared to age-matched male and female controls ( $>18,<67$ years). 
A

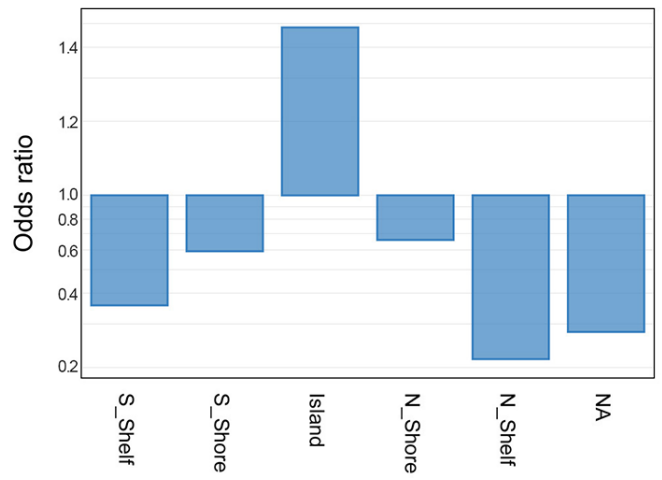

B

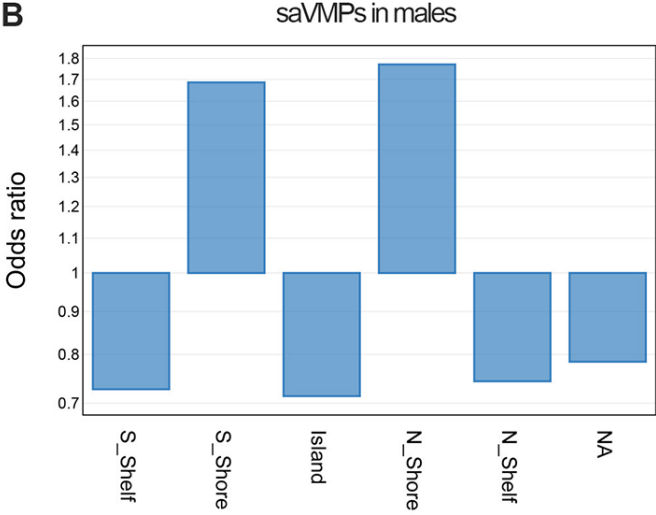

Supplementary Figure 6. Enrichment (odds ratio) of genomic localizations for ssaVMPs calculated from beta values (A) or residuals (B).
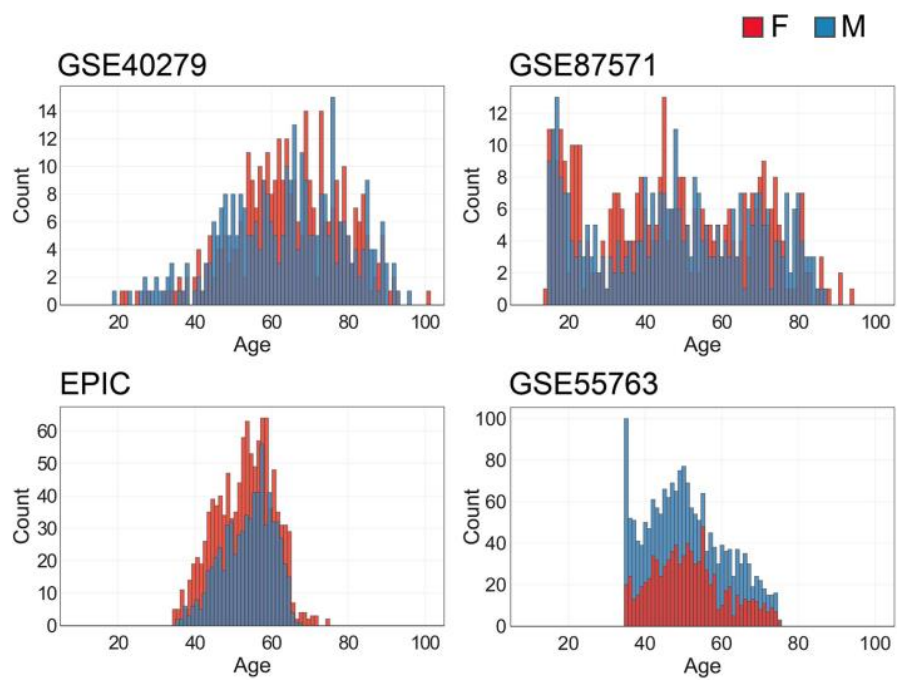

Supplementary Figure 7. Histograms of the number of females (red) and males (blue) according to age in GSE40279, GSE87571, EPIC and GSE55763 datasets. 


\section{Supplementary Tables}

Supplementary Table 1. Age-by-sex interaction in the number of epimutations and in the values of Shannon entropy. ANCOVA p-values are reported for age, sex and age-by-sex interaction effects.

\begin{tabular}{lcccccc}
\hline & \multicolumn{3}{c}{ Epimutations } & \multicolumn{3}{c}{ Entropy } \\
\cline { 2 - 7 } & age & sex & age:sex & age & sex & age:sex \\
\hline GSE40279 & $6.8093 \mathrm{E}-08$ & $4.8010 \mathrm{E}-01$ & $5.1342 \mathrm{E}-01$ & $7.7160 \mathrm{E}-01$ & $7.7393 \mathrm{E}-02$ & $3.6785 \mathrm{E}-01$ \\
GSE87571 & $9.1517 \mathrm{E}-20$ & $9.4685 \mathrm{E}-01$ & $7.2585 \mathrm{E}-01$ & $1.4711 \mathrm{E}-17$ & $1.3101 \mathrm{E}-01$ & $4.7210 \mathrm{E}-01$ \\
EPIC & $1.6369 \mathrm{E}-08$ & $5.8827 \mathrm{E}-02$ & $8.0821 \mathrm{E}-02$ & $1.3407 \mathrm{E}-04$ & $4.8294 \mathrm{E}-05$ & $1.0310 \mathrm{E}-04$ \\
GSE55763 & $1.0350 \mathrm{E}-15$ & $4.9039 \mathrm{E}-02$ & $2.6199 \mathrm{E}-02$ & $3.4323 \mathrm{E}-09$ & $3.2372 \mathrm{E}-01$ & $1.9585 \mathrm{E}-01$ \\
\hline
\end{tabular}

Supplementary Table 2. Characteristics of the Infinium450k datasets investigated in the present study.

\begin{tabular}{lcccc}
\hline & GSE40279 & GSE87571 & EPIC & GSE55763 \\
\hline Number of subjects & 656 & 729 & 1803 & 2670 \\
Number of females & 338 & 388 & 1114 & 860 \\
Number of males & 318 & 341 & 689 & 1810 \\
Age range & $19-101$ & $14-94$ & $34-74$ & $35-75$ \\
Ethnic group (Country) & European and & European (Sweden) & European (Italy) & Indian Asian and \\
& Hispanic (USA) & European (UK) \\
\hline
\end{tabular}

Supplementary Table 3. Characteristics of the samples analyzed by the EpiTYPER assay.

\begin{tabular}{lcccc}
\hline & $\begin{array}{c}\text { Healthy controls } \\
\mathbf{n = 4 1 9}\end{array}$ & $\begin{array}{c}\text { Centenarians } \\
\mathbf{n}=\mathbf{4 9}\end{array}$ & $\begin{array}{c}\text { Centenarians' offspring } \\
\mathbf{n}=\mathbf{4 8}\end{array}$ & $\begin{array}{c}\text { Down syndrome } \\
\mathbf{n}=\mathbf{4 4}\end{array}$ \\
\hline Males & $\mathrm{n}=198$ & $\mathrm{n}=15$ & $\mathrm{n}=15$ & $\mathrm{n}=25$ \\
& $25-98$ years & $100-105$ years & $58-84$ years & $22-63$ years \\
Females & $\mathrm{n}=221$ & $\mathrm{n}=34$ & $\mathrm{n}=33$ & $\mathrm{n}=19$ \\
& $23-98$ years & $100-112$ years & $55-89$ years & $19-66$ years \\
\hline
\end{tabular}




\section{Supplementary Files}

Supplementary File 1. Lists of sex- and age-associated differentially methylated positions (saDMPs) and of sex- but not age-associated differentially methylated positions (snaDMPs) resulting from the meta-analysis.

Supplementary File 2. Gene Ontology enrichment of saDMPs and snaDMPs lists.

Supplementary File 3. Probes with significant age-by-sex interaction resulting from the meta-analysis.

Supplementary File 4. Results of the statistical analyses performed on EpiTYPER data on centenarians', centenarians' offspring and Down syndrome cohorts.

Supplementary File 5. Lists of sex-specific age-associated variably methylated positions (saVMPs).

Supplementary File 6. Gene Ontology enrichment of saVMPs lists.

Supplementary File 7. Results of "Ominer" tool for FIGN, DOC2A, PEX10 and PRR4 genes.

Supplementary File 8. Imprinted regions and sex hormones-related genes considered for enrichment analysis. 